\title{
Unravelling Incommensurate Magnetism and Its Emergence in Iron-Based Superconductors
}

\author{
Morten H. Christensen, ${ }^{1}$ Brian M. Andersen, ${ }^{1}$ and Panagiotis Kotetes ${ }^{1,2}$ \\ ${ }^{1}$ Niels Bohr Institute, University of Copenhagen, Juliane Maries Vej 30, 2100 Copenhagen, Denmark \\ ${ }^{2}$ Center for Quantum Devices, Niels Bohr Institute, University of Copenhagen, \\ 2100 Copenhagen, Denmark
}

(Received 23 February 2018; revised manuscript received 22 June 2018; published 9 November 2018)

\begin{abstract}
We focus on a broad class of tetragonal itinerant systems sharing a tendency towards the spontaneous formation of incommensurate magnetism with ordering wave vectors $Q_{1,2}=(\pi-\delta, 0) /(0, \pi-\delta)$ or $\boldsymbol{Q}_{1,2}=(\pi, \delta) /(-\delta, \pi)$. Employing a Landau approach, we obtain the generic magnetic phase diagram and identify the leading instabilities near the paramagnetic-magnetic transition. Nine distinct magnetic phases exist that either preserve or violate the assumed $C_{4}$ symmetry of the paramagnetic phase. These are singleand double- $\boldsymbol{Q}$ phases consisting of magnetic stripes, helices, and whirls, either in an individual or coexisting manner. These nine phases can be experimentally distinguished by polarized neutron scattering, or, e.g., by combining measurements of the induced charge order and magnetoelectric coupling. Within two representative five-orbital models, suitable for $\mathrm{BaFe}_{2} \mathrm{As}_{2}$ and $\mathrm{LaFeAsO}$, we find that the incommensurate magnetic phases we discuss here are accessible in iron-based superconductors. Our investigation unveils a set of potential candidates for the unidentified $C_{2}$-symmetric magnetic phase that was recently observed in $\mathrm{Ba}_{1-x} \mathrm{Na}_{x} \mathrm{Fe}_{2} \mathrm{As}_{2}$. Among the phases stabilized we find a spin-whirl crystal, which is a textured magnetic $C_{4}$-symmetric phase. The possible experimental observation of textured magnetic orders in iron-based superconductors opens new directions for realizing intrinsic topological superconductors.

DOI: $10.1103 / P h y s R e v X .8 .041022$

Subject Areas: Condensed Matter Physics,
Magnetism,
Superconductivity
\end{abstract}

\section{INTRODUCTION}

Magnetism constitutes one of the most ubiquitous phases in correlated matter, giving rise to a wide range of remarkable phenomena. Apart from the typical examples of (anti) ferromagnetism, there exists an entire zoo of other magnetic phases, ranging from collinear to noncoplanar orders. The iron-based superconductors (FeSCs) are a prime example of this diversity. The commensurate magnetic stripe (MS) phase, with moments aligned antiferromagnetically along one $\mathrm{Fe}-\mathrm{Fe}$ direction and ferromagnetically along the other, is prevalent in the undoped compounds. However, recent experiments on a number of hole-doped compounds have revealed the emergence of both collinear [1] and coplanar [2] magnetic orders.

Commensurate magnetism in the FeSCs can be described by two magnetic order parameters $\boldsymbol{M}_{1}$ and $\boldsymbol{M}_{2}$, with ordering vectors $\boldsymbol{Q}_{1}=(\pi, 0)$ and $\boldsymbol{Q}_{2}=(0, \pi)$ (in the $1 \mathrm{Fe} /$ unit cell),

Published by the American Physical Society under the terms of the Creative Commons Attribution 4.0 International license. Further distribution of this work must maintain attribution to the author(s) and the published article's title, journal citation, and DOI. related by fourfold $\left(C_{4}\right)$ rotations [3-12]. In the event that the two order parameters compete, the single- $\boldsymbol{Q}$ MS phase is realized. On the other hand, if the two order parameters coexist, the moments can align in either a parallel $\left(\boldsymbol{M}_{1} \| \boldsymbol{M}_{2}\right)$ or perpendicular $\left(\boldsymbol{M}_{1} \perp \boldsymbol{M}_{2}\right)$ fashion. In the case of collinear moments, the resulting magnetic order is dubbed a chargespin density wave (CSDW) phase, while the case of noncollinear moments is denoted a spin-vortex crystal (SVC) phase. These so-called double- $Q$ phases can be challenging to distinguish experimentally, and this generally requires the use of local probe techniques. Signatures of double- $\boldsymbol{Q}$ magnetic structures were observed in $\mathrm{Ba}_{1-x} \mathrm{Na}_{x} \mathrm{Fe}_{2} \mathrm{As}_{2}$ [13,14], $\mathrm{Ba}_{1-x} \mathrm{~K}_{x} \mathrm{Fe}_{2} \mathrm{As}_{2}$ [15-20], $\mathrm{Sr}_{1-x} \mathrm{Na}_{x} \mathrm{Fe}_{2} \mathrm{As}_{2}$ [1], and in hole-doped $\mathrm{CaKFe}_{4} \mathrm{As}_{4}$ [2]. In $\mathrm{Sr}_{1-x} \mathrm{Na}_{x} \mathrm{Fe}_{2} \mathrm{As}_{2}$, Mössbauer spectroscopy captured the existence of a CSDW phase [1], while in $\mathrm{CaKFe}_{4} \mathrm{As}_{4}$ a SVC phase was observed [2]. Their discovery has generated considerable attention, and especially the observation of a CSDW phase highlights the itinerant nature of magnetism in these compounds.

Nevertheless, recent experiments suggest that magnetism in FeSCs may not only be limited to the three phases mentioned above. In fact, the commensurate aspect needs to be revisited since direct evidence for incommensurate (IC) 
TABLE I. Characteristics and experimental signatures of the nine distinct incommensurate magnetic phases, which are extrema of Eq. (1). The wave vectors appearing in the column regarding the induced charge order have been separated into groups. For a given group the Bragg peaks have the same intensity. Note that the wave vectors in light font in the column "Induced charge order," depending on the parameters, may not lead to a Bragg peak in the spectrum. $\mathcal{B}$ and $\mathcal{E}$ are Zeeman and electric fields, respectively, and $\mathcal{C}$ denotes the Skyrmion charge.

\begin{tabular}{|c|c|c|c|c|}
\hline Magnetic phase & Rotational symmetry & Induced charge order & Magnetoelectricity & $\mathcal{B}$-induced $\mathcal{C}$ \\
\hline IC MS $\left(Q_{1}\right)$ & $C_{2}$ & $\left\{ \pm 2 Q_{1}\right\}$ & $x$ & $x$ \\
\hline IC CSDW & $C_{4}$ & $\left\{ \pm 2 \boldsymbol{Q}_{1}, \pm 2 \boldsymbol{Q}_{2}, \pm \boldsymbol{Q}_{1} \pm \boldsymbol{Q}_{2}\right\}$ & $x$ & $x$ \\
\hline IC SVC & $C_{4}^{4}$ & $\left\{ \pm 2 \widehat{\boldsymbol{Q}_{1}}, \pm 2 \boldsymbol{Q}_{2}\right\}$ & $x$ & $x$ \\
\hline MH $\left(\boldsymbol{Q}_{2}\right)$ & $C_{2}$ & $x$ & $\checkmark\left(M_{0} \propto Q_{2} \cdot \mathcal{E}\right)$ & $x$ \\
\hline $\operatorname{MS}\left(\boldsymbol{Q}_{1}\right) \| \mathrm{MH}\left(\boldsymbol{Q}_{2}\right)$ & $C_{2}$ & $\left\{ \pm 2 \boldsymbol{Q}_{1}\right\},\left\{ \pm 2 Q_{2}\right\},\left\{ \pm \boldsymbol{Q}_{1} \pm \boldsymbol{Q}_{2}\right\}$ & $\checkmark\left(M_{0} \propto Q_{2} \cdot \mathcal{E}\right)$ & $x$ \\
\hline $\operatorname{MS}\left(\boldsymbol{Q}_{1}\right) \perp \mathrm{MH}\left(\boldsymbol{Q}_{2}\right)$ & $\mathrm{C}_{2}$ & $\left\{ \pm 2 \boldsymbol{Q}_{1}\right\}$ & $\checkmark\left(\boldsymbol{M}_{\mathbf{0}} \propto \boldsymbol{Q}_{2} \cdot \mathcal{E}\right)$ & $x$ \\
\hline $\mathrm{DPMH}\left(\hat{\boldsymbol{n}}_{1}=\hat{\boldsymbol{n}}_{2}\right)$ & $C_{2}$ & $\left\{ \pm\left(\boldsymbol{Q}_{1}-\boldsymbol{Q}_{2}\right)\right\}$ & $\checkmark\left(\boldsymbol{M}_{\mathbf{0}} \propto\left(\boldsymbol{Q}_{1}+\boldsymbol{Q}_{2}\right) \cdot \mathcal{E}\right)$ & $x$ \\
\hline $\mathrm{SWC}_{4}$ & $C_{4}$ & $\left\{ \pm 2 \boldsymbol{Q}_{1}, \pm 2 \boldsymbol{Q}_{2}\right\},\left\{ \pm \boldsymbol{Q}_{1} \pm \boldsymbol{Q}_{2}\right\}$ & $\checkmark$ & $\checkmark(\mathcal{C}= \pm 1)$ \\
\hline $\mathrm{SWC}_{2}\left[\hat{\boldsymbol{n}}_{1,2}\right.$ of Eq. (14)] & $C_{2}$ & $\left\{ \pm 2 \boldsymbol{Q}_{2}\right\},\left\{ \pm \boldsymbol{Q}_{1} \pm \boldsymbol{Q}_{2}\right\}$ & $\checkmark$ & $\checkmark(\mathcal{C}= \pm 1)$ \\
\hline
\end{tabular}

magnetism has been provided by neutron scattering [21-23] on electron-doped FeSCs. In addition, recent thermal expansion measurements [24] performed on hole-doped $\mathrm{Ba}_{1-x} \mathrm{Na}_{x} \mathrm{Fe}_{2} \mathrm{As}_{2}$ have revealed a rich mosaic of singleand double- $\boldsymbol{Q}$ magnetic phases, including a new $C_{2^{-}}$symmetric phase of a currently unidentified nature. Importantly, the latter experiment found evidence for the presence of an inflection point in the magnetic transition temperature as a function of doping. This has been interpreted as the onset of IC magnetic order [25,26], although the presence of IC magnetism in this compound has not yet been confirmed by scattering experiments. We note that the existence of IC magnetic order is entirely expected within an itinerant scenario for magnetism in the FeSCs.

Similar to other high- $T_{c}$ superconductors [27,28], superconductivity in the FeSCs emerges via charge doping of the parent compounds. This leads to the possibility of microscopic coexistence between superconductivity and magnetism, which has indeed been observed in certain pnictogen compounds [13,24,29-33]. In the context of textured noncollinear and noncoplanar magnetic phases, such a coexistence is of particular interest. These types of orders tie together the orbital and spin degrees of freedom and induce an effective spin-orbit coupling (SOC) [34]. When the induced SOC breaks inversion symmetry, such magnetic orders can give rise to magnetoelectric phenomena, akin to those encountered in spin-Skyrmion crystals [35], multiferroics [36], semiconductors with non-negligible SOC [37,38], and topological insulators [39]. More importantly, the simultaneous violation of time-reversal symmetry and the generation of SOC renders the aforementioned magnetic textures suitable building blocks for realizing topological superconductors (TSCs) [40-57]. This can be achieved either via proximity of these magnetic textures to a conventional superconductor, or intrinsically, by virtue of their microscopic coexistence with spin-singlet superconductivity. An intrinsic TSC remains a long-sought-after but also elusive phase of matter, and accomplishing it via the above mechanism is challenging due to the antagonistic relation of magnetic order and spin-singlet superconductivity.

Motivated by the recent experimental developments and the possible applications for TSCs, we explore the generic consequences of an IC magnetic ordering vector. Interestingly, we find that this leads to a number of magnetic phases distinct from the previously discussed stripe, collinear, and noncollinear orders. For this purpose, we first carry out a systematic exploration of the accessible IC magnetic orders with wave vectors $\boldsymbol{Q}_{1,2}=(\pi-\delta, 0) /$ $(0, \pi-\delta)$ or $Q_{1,2}=(\pi, \delta) /(-\delta, \pi)$. Within a generic Landau approach we identify nine possible IC magnetic ground states, and subsequently extract the phase diagram. The appearance of new phases demonstrates that the effect of incommensurability is not limited to a simple generalization of the three known commensurate phases. Among the phases we find a single- $Q$ magnetic helix $(\mathrm{MH})$ phase along with two double- $Q$ phases where an IC stripe coexists with a magnetic helix. Additionally, we find a double- $\boldsymbol{Q}$ phase consisting of two parallel helices, and, finally, two double- $\boldsymbol{Q}$ phases constituting, as coined here, the spinwhirl crystals. The fundamental properties of all the nine magnetic phases are summarized in Table I, while the respective spatial profiles of the magnetization are depicted in Figs. 1 and 2, and discussed in greater detail below.

While the IC scenario provides a number of candidates for the unidentified magnetic phase appearing in $\mathrm{Na}$-doped $\mathrm{BaFe}_{2} \mathrm{As}_{2}$, an unambiguous identification requires additional experimental measurements. In order to identify and distinguish these nine magnetic ground states and their possible presence in FeSCs, one can employ a combination of experimental techniques capable of resolving the magnetically induced charge order in the system and the emergence of a nonzero magnetoelectric coupling. The former can be detected in x-ray scattering and the emerging Bragg peaks. For the latter, one can measure the magnetization generated by applying an external electric field $(\mathcal{E})$ or via inducing a current $(\boldsymbol{I})$ flow. We predict that a 
(a)

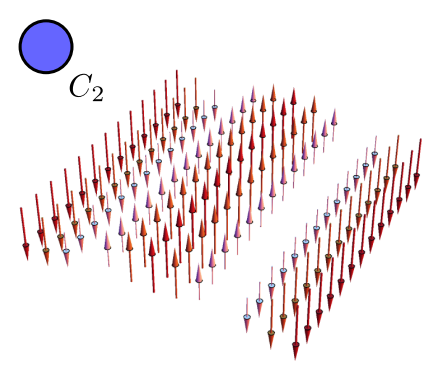

(b)



(c)

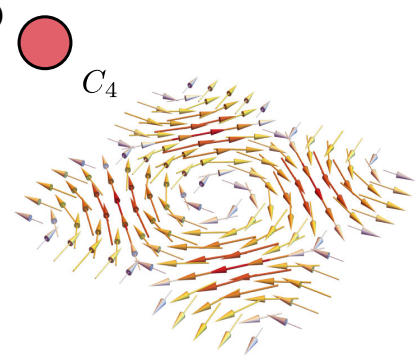

FIG. 1. Illustration of the IC generalization of the three well-known commensurate magnetic phases with ordering wave vectors $(0, \pi)$ and $(\pi, 0)$. Here we restrict to a single magnetic unit cell and depict the spatial profile of the magnetization for (a) the $C_{2}$-symmetric IC magnetic stripe (IC-MS) phase, (b) the $C_{4}$-symmetric IC charge-spin density wave (IC-CSDW) phase, and (c) the $C_{4}$-symmetric IC spinvortex crystal (IC-SVC) phase.

homogeneous magnetization can appear in the presence of helices or whirls in the magnetic ground state, depending on the orientation of $\mathcal{E}$ or $\boldsymbol{I}$. Our results regarding the experimental signatures of the nine magnetic phases are summarized in Table I.

By adopting two five-orbital models [58,59] based on density functional theory (DFT) calculations for $\mathrm{BaFe}_{2} \mathrm{As}_{2}$ and $\mathrm{LaFeAsO}$, respectively, we demonstrate that IC magnetism is a realistic scenario for the family of FeSCs. Remarkably, textured magnetic phases are accessible for both band structure types considered here. In fact, $\mathrm{BaFe}_{2} \mathrm{As}_{2}$ supports a variety of textured phases. On the other hand, textured phases can be stabilized in $\mathrm{LaFeAsO}$ by taking into account orbital selectivity [60-63], modeled in the present case with orbital-dependent interactions. For the explored parameter regime, we find that a
$C_{4}$-symmetric spin-whirl crystal phase becomes favored in both cases. Remarkably, this can acquire a topologically nontrivial Skyrmion charge by the application of a weak Zeeman field $(\mathcal{B})$.

The possible emergence of new magnetic phases in FeSCs does not only add to the established commensurate picture regarding the magnetic phase diagram, it also opens up perspectives for crafting novel topological phases. In fact, the experimentally confirmed microscopic coexistence of magnetism and superconductivity in FeSCs $[13,24,32,33]$ appears as a promising platform for realizing intrinsic TSCs. While symmetry constraints [44] prohibit crafting strong TSCs [39] by solely combining the standard commensurate magnetic phases with spin-singlet superconductivity, the possible emergence of noncoplanar magnetic textures could provide a way around this obstacle, (a)
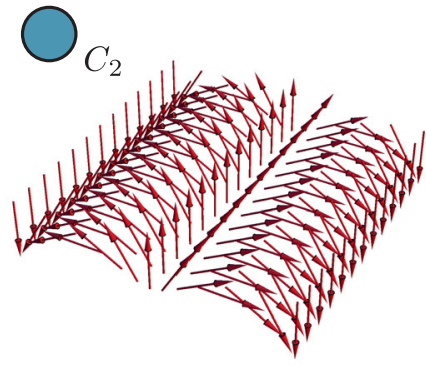

(d)

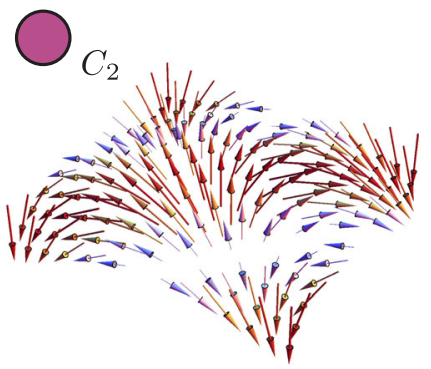

(b)

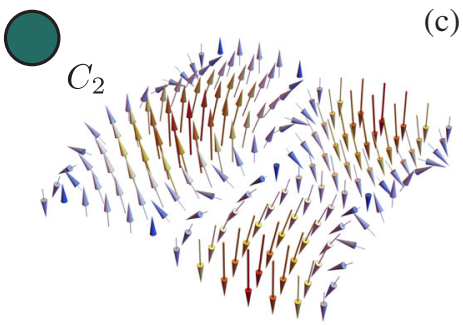

(e)

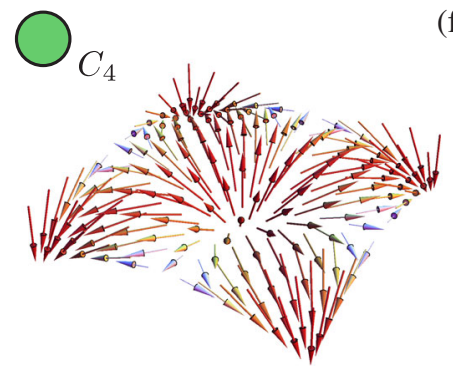

(c)<smiles>[GeH3]</smiles>



(f)

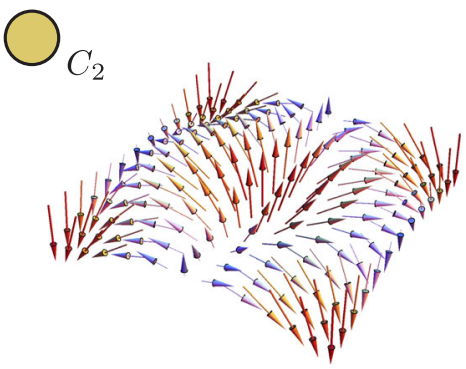

FIG. 2. Novel magnetic phases appearing with the rise of IC magnetism. The color scale signifies the magnitude of the magnetic moment. Here we restrict to a single magnetic unit cell. Contrary to the commensurate case, the incommensurability allows for noncoplanar magnetic textures. In panels (a)-(c) various magnetic helix (MH) order parameters are shown. In panels (b) and (c) the helix coexists with an in-plane or out-of-plane IC stripe (MS $\| \mathrm{MH}$ or $\mathrm{MS} \perp \mathrm{MH})$. In panel (d) we present the $C_{2}$-symmetric double parallel magnetic helix (DPMH) phase, and in panels (e) and (f) the noncoplanar spin-whirl crystal $\left(\mathrm{SWC}_{4}\right.$ or $\left.\mathrm{SWC}_{2}\right)$ phase with $C_{4}$ or $C_{2}$ symmetry. 
as previous proposals on artificial chiral topological SCs [44,45,53-57] suggest. We note that the magnetically engineered TSCs relevant here are distinct from standard SOC-generated TSCs which may also be relevant in some of the FeSCs, as revealed by recent angle-resolved photoemission spectroscopy and scanning tunneling microscopy experiments $[64,65]$.

The content of this paper is organized as follows. In Sec. II, we present the general Landau formalism and discuss the emergence of nine IC magnetic phases, among which one finds a number of potential candidates for the recently discovered phase in $\mathrm{Ba}_{1-x} \mathrm{Na}_{x} \mathrm{Fe}_{2} \mathrm{As}_{2}$ [24]. The detailed magnetic structures of these nine phases are presented in Sec. III. In Sec. IV, we show the generic magnetic phase diagram, illustrating the leading magnetic instability as a function of the Landau parameters. Section V discusses experimental signatures of the IC phases, focusing on how to distinguish them from one another without relying on, e.g., detailed polarized neutron scattering measurements. In Sec. VI, we turn to FeSCs and derive the Landau functional for the two microscopic five-band models, and include interactions in a mean-field manner using a standard multiorbital Hubbard-Hund Hamiltonian. This allows for a microscopic determination of the Landau coefficients, the thermodynamic magnetic phase diagram, and, subsequently, an analysis of the energetics of the IC magnetic phases. Moreover, we discuss the effects of the inversion-symmetric atomic SOC on the magnetic phase diagram and the appearance of noncoplanar magnetic textures. Finally, Sec. VII summarizes our results and discusses further directions.

\section{LANDAU FORMALISM FOR INCOMMENSURATE MAGNETISM}

To identify the accessible IC magnetic phases for a generic itinerant system with tetragonal symmetry and ordering wave vectors $\boldsymbol{Q}_{1,2}=(\pi-\delta, 0) /(0, \pi-\delta)$ or $\boldsymbol{Q}_{1,2}=(\pi, \delta) /(-\delta, \pi)$, we write down the Landau functional up to quartic order with respect to the magnetic order parameters $\boldsymbol{M}\left(\boldsymbol{Q}_{1,2}\right) \equiv \boldsymbol{M}_{1,2}$. Contrary to the commensurate case $(\delta=0)$, where $\boldsymbol{M}_{1,2}=\boldsymbol{M}_{1,2}^{*}$, in the present IC situation the magnetic order parameters are complex since $\boldsymbol{M}\left(\boldsymbol{Q}_{1,2}\right) \neq \boldsymbol{M}\left(\boldsymbol{Q}_{1,2}\right)^{*} \equiv \boldsymbol{M}\left(-\boldsymbol{Q}_{1,2}\right)$. This results in the following expression for the Landau free-energy functional:

$$
\begin{aligned}
F= & \alpha\left(\left|\boldsymbol{M}_{1}\right|^{2}+\left|\boldsymbol{M}_{2}\right|^{2}\right)+\frac{\tilde{\beta}}{2}\left(\left|\boldsymbol{M}_{1}\right|^{2}+\left|\boldsymbol{M}_{2}\right|^{2}\right)^{2} \\
& +\frac{\beta-\tilde{\beta}}{2}\left(\left|\boldsymbol{M}_{1}^{2}\right|^{2}+\left|\boldsymbol{M}_{2}^{2}\right|^{2}\right)+(g-\tilde{\beta})\left|\boldsymbol{M}_{1}\right|^{2}\left|\boldsymbol{M}_{2}\right|^{2} \\
& +\frac{\tilde{g}}{2}\left(\left|\boldsymbol{M}_{1} \cdot \boldsymbol{M}_{2}\right|^{2}+\left|\boldsymbol{M}_{1} \cdot \boldsymbol{M}_{2}^{*}\right|^{2}\right) .
\end{aligned}
$$

The above was previously studied by Schulz [66], restricted, however, to the possible occurrence of IC magnetism in high- $T_{\mathrm{c}}$ cuprates. We note that the free energy given in Eq. (1) is obtainable from a microscopic electronic model, as we demonstrate in Sec. VI.

The Landau functional in Eq. (1) is invariant under complex conjugation $(\mathcal{K})$, time reversal $(\mathcal{T}), D_{4 h}$ point group operations, $\mathrm{SO}(3)$ spin rotations, and translations $\left(t_{\boldsymbol{a}}\right.$, with $\boldsymbol{a}$ the direct lattice shift vector). At quadratic level the above symmetry becomes artificially enhanced, yielding a degeneracy among the possible candidate phases for the leading magnetic instability that sets in when $\alpha<0$. The latter guides us to parametrize the order parameters by

$$
\boldsymbol{M}_{1}=M \cos \eta \hat{\boldsymbol{n}}_{1} \quad \text { and } \quad \boldsymbol{M}_{2}=M \sin \eta \hat{\boldsymbol{n}}_{2},
$$

with $\left|\hat{\boldsymbol{n}}_{1,2}\right|^{2}=1$ and $\eta \in[0, \pi / 2]$. Note that the complex spin vectors generally satisfy $\left|\hat{\boldsymbol{n}}_{1,2}^{2}\right| \leq 1$. Translational invariance allows us to arbitrarily and independently choose the overall phase of the vectors $\hat{\boldsymbol{n}}_{1,2}$. On the other hand, spin-rotational invariance allows for further simplifications, for instance setting $\operatorname{Re}\left[\hat{\boldsymbol{n}}_{1}\right]$ parallel to the $z$ spin axis. Note that this is not possible if SOC is present since this introduces anisotropy in spin space. The effects of a weak SOC are discussed in Sec. VIC.

Under these conditions, extremizing the Landau functional with respect to $\eta$ yields

$$
\sin (2 \eta)=0, \quad \cos (2 \eta)=\frac{\left|\hat{\boldsymbol{n}}_{1}^{2}\right|^{2}-\left|\hat{\boldsymbol{n}}_{2}^{2}\right|^{2}}{2 G+2 \tilde{G} P-\left(\left|\hat{\boldsymbol{n}}_{1}^{2}\right|^{2}+\left|\hat{\boldsymbol{n}}_{2}^{2}\right|^{2}\right)},
$$

where we have introduced

$$
G \equiv \frac{g-\tilde{\beta}}{\beta-\tilde{\beta}}, \quad \tilde{G} \equiv \frac{\tilde{g}}{\beta-\tilde{\beta}}, \quad P \equiv \frac{\left|\hat{\boldsymbol{n}}_{1} \cdot \hat{\boldsymbol{n}}_{2}\right|^{2}+\left|\hat{\boldsymbol{n}}_{1} \cdot \hat{\boldsymbol{n}}_{2}^{*}\right|^{2}}{2}
$$

For $\sin (2 \eta)=0$, we retrieve single- $Q$ phases since $\eta=0$ $(\eta=\pi / 2)$ implies that only the order parameter with wave vector $\boldsymbol{Q}_{1}\left(\boldsymbol{Q}_{2}\right)$ appears.

The remaining extrema arise for values of $\eta$ determined by $\cos (2 \eta)$, leading to double- $\boldsymbol{Q}$ phases. For $\left|\hat{\boldsymbol{n}}_{1}^{2}\right| \neq\left|\hat{\boldsymbol{n}}_{2}^{2}\right|$, we have $\eta \neq \pi / 4$. By observing that $\tan \eta=\left|\boldsymbol{M}_{2}\right| /\left|\boldsymbol{M}_{1}\right|$, we obtain that in this case $\left|\boldsymbol{M}_{1}\right| \neq\left|\boldsymbol{M}_{2}\right|$ and, thus, all the arising double- $Q$ phases violate $C_{4}$ symmetry, leaving only a $C_{2}$ subgroup intact. If instead $\left|\hat{\boldsymbol{n}}_{1}^{2}\right|=\left|\hat{\boldsymbol{n}}_{2}^{2}\right|$, not necessarily all double- $Q$ phases are $C_{4}$-symmetry violating. After excluding special or singular values of the Landau coefficients, we find nine extrema of the free energy. These are presented in Table I and in Sec. III. Further details regarding the corresponding free energy are presented in Appendix A. Finally, note that the two possible values for the sign of $\beta-\tilde{\beta}$ give rise to two generic phase diagrams that we present in detail in Sec. IV. 


\section{INCOMMENSURATE MAGNETIC PHASES}

The order parameters of the accessible magnetic phases are identified by the values of $\eta$ and the spin vectors $\hat{\boldsymbol{n}}_{1,2}$, which are determined by minimizing the free energy of Eq. (1). In addition to the IC extensions of the three wellknown commensurate single- $\boldsymbol{Q}$ stripe (MS) and double- $\boldsymbol{Q}$, collinear (CSDW) and noncollinear (SVC) phases, we uncover a single- $Q$ helix phase along with five further double- $\boldsymbol{Q}$ phases. These double- $\boldsymbol{Q}$ phases include a number of $C_{4}$-symmetry breaking phases.

\section{A. IC extensions of the known commensurate magnetic phases}

We commence by considering the IC generalizations of the three well-studied commensurate phases, the $C_{2}$-symmetric MS phase along with the $C_{4}$-symmetric CSDW and SVC phases.

IC magnetic stripe (IC MS).- - This constitutes the generalization of the typical magnetic $C_{2}$-symmetric phase, prevalent in FeSCs. As a single- $\boldsymbol{Q}$ phase, it can appear for either $\boldsymbol{Q}_{1}$ or $\boldsymbol{Q}_{2}$. Since the latter are equivalent, they can lead to domains in actual materials. Throughout this work we focus on single-domain realizations and choose the magnetic ordering vector of the IC MS to be $Q_{1}=(\pi-\delta, 0)$ throughout. Thus we obtain

$$
\hat{\boldsymbol{n}}_{1}=(0,0,1) \quad \text { and } \quad \hat{\boldsymbol{n}}_{2}=(0,0,0),
$$

which yields the magnetic order depicted in Fig. 1(a).

IC charge-spin density wave (IC CSDW).-This is a double- $Q C_{4}$-symmetric phase which constitutes the IC extension of the CSDW phase and can be seen as two superimposed IC-MS phases with parallel magnetic-orderparameter spin vectors. An example of this magnetic order with $\boldsymbol{Q}_{1,2}=(\pi-\delta, 0) /(0, \pi-\delta)$ and

$$
\hat{\boldsymbol{n}}_{1}=(0,0,1) \quad \text { and } \quad \hat{\boldsymbol{n}}_{2}=(0,0,1)
$$

is shown in Fig. 1(b).

IC spin-vortex crystal (IC SVC).-This generalizes the commensurate SVC phase. It is a double- $Q C_{4}$-symmetric phase, and similarly to the IC-CSDW phase above, it can be viewed as two MS phases superimposed. The difference in this case is that the spin vectors of the magnetic order parameter are oriented in a perpendicular fashion. An example of such a magnetic order with $\boldsymbol{Q}_{1,2}=(\pi-\delta, 0) /$ $(0, \pi-\delta)$ is

$$
\hat{\boldsymbol{n}}_{1}=(0,1,0) \quad \text { and } \quad \hat{\boldsymbol{n}}_{2}=(1,0,0) .
$$

This phase is depicted in Fig. 1(c). It is worth commenting on the nomenclature of the particular magnetic phase, which reflects the presence of singular points in the spatial profile of the magnetization. The singular points act as sources of vorticity, cf. Refs. [67,68]. This becomes apparent by rewriting the here two-dimensional magnetization vector as follows: $\boldsymbol{M}(\boldsymbol{r})=\left(M_{x}(\boldsymbol{r}), M_{y}(\boldsymbol{r}), 0\right) \equiv$ $|\boldsymbol{M}(\boldsymbol{r})|(\cos \varphi(\boldsymbol{r}), \sin \varphi(\boldsymbol{r}), 0)$. The vorticity sources are located at points in space for which $|\boldsymbol{M}(\boldsymbol{r})|=0$ and for which at the same time the phase field $\varphi(\boldsymbol{r})$ jumps by $n$ multiples of $2 \pi$ as we move around a loop encircling the singularity. Here $n$ corresponds to the number of vorticity units carried by the defect. For the choice of spin vectors in Eq. (7), the coordinates for the vorticity sources are given by $\boldsymbol{r}_{s}=[\pi /(2 Q)]( \pm 1, \pm 1)$. The vorticity points are also depicted in Fig. 1(c), where we have chosen the unit cell such that it contains one of the vortices located at the center. One finds that each singular point contributes with a single unit of vorticity, while within a single magnetic unit cell the total vorticity is zero.

\section{B. New magnetic phases arising by virtue of incommensurability}

Apart from the anticipated IC extensions of the three wellknown commensurate magnetic phases encountered in the FeSCs, we find additional phases unique to the IC case. These are portrayed in Figs. 2(a)-2(f). In Fig. 2(a) we present a single- $Q$ magnetic helix phase. In Figs. 2(b) and 2(c) the magnetic helix phase coexists with an in-plane or out-ofplane magnetic spiral. In Fig. 2(d) a $C_{2}$-symmetric double parallel $\mathrm{MH}(\mathrm{DPMH})$ phase is shown, while in Fig. 2(e) a noncoplanar $C_{4}$-symmetric spin-whirl crystal $\left(\mathrm{SWC}_{4}\right)$ phase is depicted. $\mathrm{A} C_{2}$ version $\left(\mathrm{SWC}_{2}\right)$ of this phase is shown in Fig. 2(f).

Magnetic helix $(M H)$.- This is a single- $Q C_{2}$-symmetric phase. As we are in a position to arbitrarily choose the ordering wave vector, below we present the spin-vector structure for the $\boldsymbol{Q}_{1}$ ordering wave vector:

$$
\hat{\boldsymbol{n}}_{1}=\frac{1}{\sqrt{2}}(i, 0,1) \quad \text { and } \quad \hat{\boldsymbol{n}}_{2}=(0,0,0) .
$$

For $\boldsymbol{Q}_{1}=(\pi-\delta, 0)$ the magnetic texture is depicted in Fig. 2(a). Note that this is the only IC magnetic phase for which the modulus of the magnetization in coordinate space $|\boldsymbol{M}(\boldsymbol{r})|$ is spatially constant.

Magnetic stripe with parallel magnetic helix (MS $\| \mathrm{MH})$ : This double- $Q C_{2}$-symmetric phase consists of a $\mathrm{MH}$ with ordering wave vector $\boldsymbol{Q}_{1(2)}$ and an IC MS with wave vector $\boldsymbol{Q}_{2(1)}$. The magnetic moment arising from the MS is oriented inside the winding plane of the $\mathrm{MH}$. By choosing one of the above allowed and equivalent wave vector configurations, the spin vectors are given by

$$
\hat{\boldsymbol{n}}_{1}=(0,0,1) \quad \text { and } \quad \hat{\boldsymbol{n}}_{2}=(i \sin \lambda, 0, \cos \lambda),
$$

and the magnetic order is shown in Fig. 2(b) for $\boldsymbol{Q}_{1,2}=$ $(\pi-\delta, 0) /(0, \pi-\delta)$. Note that the parameter $\lambda$ is provided by minimizing the free energy and its expression is presented in Appendix A. 
Magnetic stripe with perpendicular magnetic helix (MS $\perp \mathrm{MH}$ ). - This double- $Q C_{2}$-symmetric phase is composed by a MH and an IC MS with magnetic moment of the stripe oriented out of the winding plane defined by the $\mathrm{MH}$. The respective magnetic vectors read

$$
\hat{\boldsymbol{n}}_{1}=(0,0,1) \quad \text { and } \quad \hat{\boldsymbol{n}}_{2}=\frac{1}{\sqrt{2}}(i, 1,0),
$$

and for $\boldsymbol{Q}_{1,2}=(\pi-\delta, 0) /(0, \pi-\delta)$ the order is depicted in Fig. 2(c). Note that this is an example of a noncoplanar magnetic order.

Double parallel magnetic helix (DPMH).-This is a double- $\boldsymbol{Q}$ coplanar $C_{2}$-symmetric phase (in spite of $\eta=\pi / 4$ ) consisting of two helices ordered at $\boldsymbol{Q}_{1,2}$, with spin winding within the same spin plane. This phase is described by identical spin vectors:

$$
\hat{\boldsymbol{n}}_{1,2}=\frac{1}{\sqrt{2}}(i, 0,1) .
$$

For $\boldsymbol{Q}_{1,2}=(\pi-\delta, 0) /(0, \pi-\delta)$ the order is shown in Fig. 2(d).

$C_{4}$-symmetric spin-whirl crystal $\left(\mathrm{SWC}_{4}\right)$. - This is a double- $Q C_{4}$-symmetric textured phase with

$$
\begin{aligned}
& \hat{\boldsymbol{n}}_{1}=(i \cos \lambda, 0, \sin \lambda), \\
& \hat{\boldsymbol{n}}_{2}=(0, i \cos \lambda, \sin \lambda) .
\end{aligned}
$$

For $\boldsymbol{Q}_{1,2}=(\pi-\delta, 0) /(0, \pi-\delta)$ we obtain the coordinate space magnetization profile:

$$
\boldsymbol{M}(\boldsymbol{r})=\sqrt{2} M\left(\begin{array}{c}
\cos \lambda \sin \left(\boldsymbol{Q}_{1} \cdot \boldsymbol{r}\right) \\
\cos \lambda \sin \left(\boldsymbol{Q}_{2} \cdot \boldsymbol{r}\right) \\
\sin \lambda\left[\cos \left(\boldsymbol{Q}_{1} \cdot \boldsymbol{r}\right)+\cos \left(\boldsymbol{Q}_{2} \cdot \boldsymbol{r}\right)\right]
\end{array}\right),
$$

plotted in Fig. 2(e) for a specific choice of $\lambda$, whose expression is given in Appendix A.

Similar to the IC-SVC phase, also the present spatial profile of the magnetization contains singular points acting as sources of topological charge located at $\boldsymbol{r}=\boldsymbol{r}_{s}$. The difference is that, in contrast to the IC-SVC case, here the magnetization is a three-dimensional vector in spin space. Thus, instead of vorticity, which characterizes singularities of a coplanar magnetic texture, we can employ the singular Berry curvature [69] $\left[\Omega_{x y}^{s}(\boldsymbol{r})=\pi \delta\left(\boldsymbol{r}-\boldsymbol{r}_{s}\right)\right]$ or $\pi$-Berry flux $[67,68,70,71]$ for assigning topological charge to the point singularities for a noncoplanar magnetic texture [71]. This explains the reasoning behind naming the particular magnetic textures as spin whirls. Note that if we focus on the vicinity of the singular points, the vorticity and the $\pi$-Berry flux coincide since the texture becomes effectively coplanar [72]. Importantly, one should not confuse the spin whirls discussed here with spin Skyrmions [67]. In the latter case the magnetic profile contains no point singularities, the Berry curvature [73] $\Omega_{x y}(\boldsymbol{r})$ can be defined for all $\boldsymbol{r}$, and the Skyrmion charge is nonzero. For further details, see Sec. V C.

For the profile of Eq. (13), the singular points are located at $(\pi / Q, 0)$ and $(0, \pi / Q)$, as given by the zeros of the modulus $|\boldsymbol{M}(\boldsymbol{r})|$. Each one of the two nonequivalent socalled nodal points contributes with a unit of topological charge. These nodes can be gapped out by either violating the $C_{4}$ symmetry or the antiunitary symmetry $\Theta=$ $\mathcal{T} t_{(\pi / Q, \pi / Q)}$, consisting of the successive operation of time reversal $\mathcal{T}$ and a direct-lattice translation by $(\pi / Q, \pi / Q)$ (see also Ref. [74]). We find $\Theta^{2}=-1$, which implies that nodal points of unit topological charge come in pairs, or that the Skyrmion charge is zero if the nodes are gapped out by breaking $C_{4}$ symmetry, cf. the next paragraph. Any type of a bulk perturbation that does not respect the above $\Theta$ symmetry lifts these nodes, thus resulting in a nonzero Berry curvature in the entire magnetic unit cell. Depending on the perturbation, the latter symmetry breaking can endow the SWC with nonzero Skyrmion charge as discussed in Sec. V C.

$C_{2}$-symmetric spin-whirl crystal $\left(S W C_{2}\right)$.- This phase constitutes a twofold symmetric version of the one above. It consists of an isotropic helix for $\boldsymbol{Q}_{1,(2)}$ coexisting with an anisotropic helix for $\boldsymbol{Q}_{2,(1)}$; i.e.,

$$
\hat{\boldsymbol{n}}_{1}=\frac{1}{\sqrt{2}}(i, 0,1) \quad \text { and } \quad \hat{\boldsymbol{n}}_{2}=(0, i \sin \lambda, \cos \lambda),
$$

which for a choice of $\lambda$ is depicted in Fig. 2(f). For the same type of IC magnetic ordering, as discussed for $\mathrm{SWC}_{4}$, we find that the nodal points are gapped out due to the broken $C_{4}$ symmetry. Nevertheless, the $\Theta$ symmetry is still present and leads to a zero Skyrmion charge.

\section{GENERIC MAGNETIC PHASE DIAGRAMS}

Having presented the allowed leading magnetic instabilities and related order parameters of the generic Landau theory of Eq. (1), we now show the generic phase diagram with respect to the rescaled parameters $g-\tilde{\beta}$ and $\tilde{g}$. We consider two cases, depending on the sign of $\beta-\tilde{\beta}$. The resulting phase diagrams are presented in Fig. 3. The $\beta-$ $\tilde{\beta}<0$ case is very reminiscent of the commensurate phase diagram, while the case with $\beta-\tilde{\beta}>0$ exhibits four out of the six new phases alongside the IC CSDW. In general, we find that the MH, IC-MS, and IC-CSDW phases dominate in a large part of the phase diagrams.

For $\beta-\tilde{\beta}<0$, only the three IC extensions of the commensurate phases appear. This resembles the wellknown commensurate phase diagram of Refs. [3,8,11]. For $\beta-\tilde{\beta}>0$, the $\mathrm{MH}$ appears for a wide range of values of the Landau parameters. In the lower right corner of Fig. 3(b), we find both the MS $\perp \mathrm{MH}$ phase and the $\mathrm{SWC}_{4}$ phase, 


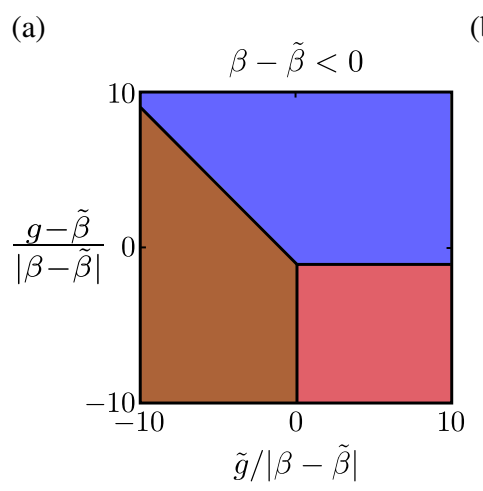

(b)

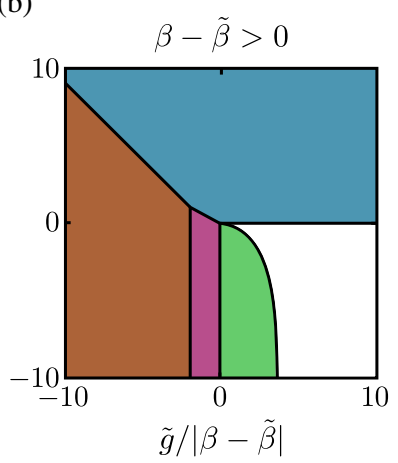

FIG. 3. Phase diagrams for the generic Landau functional in Eq. (1), where $\beta-\tilde{\beta}<0$ (a) and $\beta-\tilde{\beta}>0$ (b), with $C_{2}$-symmetric IC magnetic stripe (IC MS) (blue filled circle), $C_{4}$-symmetric IC charge-spin density wave (IC-CSDW) phase (brown circle), $C_{4}$-symmetric IC spin-vortex crystal (IC-SVC) phase (pink circle), $C_{2}$-symmetric magnetic helix $(\mathrm{MH})$ (blue filled circle), $C_{2}$-symmetric IC magnetic stripe with a perpendicular $(\perp)$ magnetic helix $(\mathrm{MS} \perp \mathrm{MH})$ phase (white), $C_{2}$-symmetric double parallel magnetic helix (DPMH) (purple filled circle), $C_{4}$-symmetric spin-whirl crystal $\left(\mathrm{SWC}_{4}\right)$ phase (green filled circle). The IC extensions of the original three phases (blue, brown, pink filled circle) are the only ones present when $\beta-\tilde{\beta}<0$, however, for $\beta-\tilde{\beta}>0$, four of the new phases occupy a substantial region of the phase diagram.

while the DPMH phase is confined to a thin sliver for negative $g-\tilde{\beta}$ and $\tilde{g}$. The remaining part of the phase diagram is occupied by the IC-CSDW phase.

Comparing the two phase diagrams reveals that the IC CSDW occupies almost the same region in both, while the IC MS is replaced by the $\mathrm{MH}$ as $\beta-\tilde{\beta}$ changes sign. For $\beta-\tilde{\beta}>0$, the DPMH and $\mathrm{SWC}_{4}$ phases share a common boundary defined by the critical line $\tilde{g} /|\beta-\tilde{\beta}|=0$, where a degeneracy among phases with an arbitrary relative orientation of the spin vectors $\boldsymbol{n}_{1,2}$ occurs. In the lower right corner, the MS $\perp$ MH phase replaces the IC SVC as $\beta-\tilde{\beta}$ becomes positive. One further observes a certain degree of similarity between the two phase diagrams. Since the phase diagram for $\beta-\tilde{\beta}<0$ resembles the commensurate one, it is useful to consider the commensurate limit $(\pi, 0)$ and $(0, \pi)$ of the IC magnetic wave vectors $\boldsymbol{Q}_{1,2}$, for the textured phases. One obtains $\mathrm{MH} \rightarrow \mathrm{MS}, \quad \mathrm{MS} \perp \mathrm{MH} \rightarrow \mathrm{SVC}$, $\mathrm{DPMH} \rightarrow \mathrm{CSDW}$ and $\mathrm{SWC}_{4} \rightarrow \mathrm{SVC}$ [75]. The latter limits provide a clear hint regarding the expected position of each textured phase in Fig. 3(b), by virtue of its continuity to the respective commensurate order.

The $\mathrm{SWC}_{2}$ and $\mathrm{MS} \| \mathrm{MH}$ do not appear as free-energy minima near the paramagnetic-magnetic transition in the parameter regime studied here. Based on the structure of the generic phase diagrams, we can conjecture that this holds in the entire parameter space. However, this statement is challenging to prove using the analytical expressions discussed in Appendix A. Therefore, we will still consider the appearance of these phases as a possible scenario. A reason to leave such a possibility open is that, since they constitute extrema of the free energy, they may still appear as metastable phases. Even more, these two phases may become thermodynamically stable under the additional presence of external fields or other more general conditions not taken into account in this study.

Finally, we remark that for the values of $g, \tilde{g}, \beta$, and $\tilde{\beta}$ considered here, the free energy is bounded from below and we can truncate the free-energy expansion at quartic order. Otherwise, one should include appropriate contributions from higher-order terms.

\section{SIGNATURES AND EXPERIMENTAL DETECTION}

From the previous section it is clear that several IC magnetic phases may become accessible, and therefore it is desirable to identify ways to differentiate them experimentally. While directly probing the spatial profile of the magnetization using spin-resolved means can certainly uniquely identify them, it can be a formidable experimental task. Thus, it is important to elaborate on alternative detection methods that do not rely on the texture's profile alone, but can still provide an unambiguous identification of the stabilized magnetic order. For this purpose we consider the combination of charge and magnetoelectric measurements. Below we provide more details regarding these two, while we additionally discuss further interesting features of the $\mathrm{SWC}_{4,2}$ phases, i.e., that they can be converted into spin-Skyrmion crystal phases via the application of an external magnetic field.

\section{A. Induced charge order}

A common characteristic of almost all the IC magnetic phases is that the respective modulus of the magnetization profile $|\boldsymbol{M}(\boldsymbol{r})|$ is not constant, but rather spatially varying. The only exception is the MH phase. As a consequence of this spatial inhomogeneity, charge order is induced when IC magnetism occurs. For a general magnetic profile with Fourier decomposition $\boldsymbol{M}(\boldsymbol{r})=\sum_{q} \boldsymbol{M}_{q} e^{i \boldsymbol{q} \cdot \boldsymbol{r}}$, the induced charge order $\rho(\boldsymbol{r})=\sum_{q} \rho_{q} e^{i q \cdot r}$ has Fourier components proportional to the scalar product of the magnetic order parameters' spin vectors $\left(\boldsymbol{M}_{q}=\left|\boldsymbol{M}_{q}\right| \hat{\boldsymbol{n}}_{q}\right)$ :

$$
\rho_{q+p} \propto\left|\boldsymbol{M}_{q}\right|\left|\boldsymbol{M}_{p}\right| \hat{\boldsymbol{n}}_{q} \cdot \hat{\boldsymbol{n}}_{\boldsymbol{p}} .
$$

Note that the above are the components generated at lowest order with respect to the magnetic order parameters, while contributions of higher order will lead to weaker effects (see also Ref. [76]). Given that here the IC magnetic ordering is considered to occur at $\boldsymbol{Q}_{1,2}$, Eq. (15) implies that there exists a set of eight possible induced components at lowest order, $\rho_{\boldsymbol{q}}$, with $\boldsymbol{q}=\left\{ \pm 2 \boldsymbol{Q}_{1}, \pm 2 \boldsymbol{Q}_{2}, \pm \boldsymbol{Q}_{1} \pm \boldsymbol{Q}_{2}\right\}$. The magnetic-charge order coupling, leading to Eq. (15), is derived in Appendix B. 
Based on Sec. III we can directly infer the Fourier components of the induced charge order for the different cases. In Table I, we display the resulting induced charge density components depending on the magnetic order. First of all, one observes that the MH, IC-SVC, and DPMH phases can be unambiguously identified via solely using the electronic charge density's Bragg peaks. The remaining six phases are split into three subsets: $\{\mathrm{IC}$ MS, MS $\perp \mathrm{MH}\}$, $\left\{\mathrm{IC} \mathrm{CSDW}, \mathrm{SWC}_{4}\right\}$, and $\left\{\mathrm{MS} \| \mathrm{MH}, \mathrm{SWC}_{2}\right\}$. The magnetic phases belonging to the same subset are precisely or practically indistinguishable via this particular experimental approach. However, the magnetic phases of each subset can be discerned based on their different magnetoelectric properties, which are discussed in the next section.

Before concluding this section, we point out that detecting charge order is more suitable for identifying IC, rather than commensurate, magnetic orders in FeSCs. This is because both commensurate magnetic ordering wave vectors $(\pi, 0)$ and $(0, \pi)$, defined in the $1 \mathrm{Fe} /$ unit cell, translate to $(\pi, \pi)$ in the $2 \mathrm{Fe} / \mathrm{unit}$ cell. In the latter, physical, unit cell, commensurate magnetism induces a charge order Bragg peak at $(0,0)$, which coincides with a lattice Bragg peak. However, for IC magnetism, the charge order is also IC and the peaks are shifted away from $(0,0)$ and should, in principle, be detectable.

\section{B. Magnetoelectric effects}

The textured nature of the magnetic phases accessible through incommensurability opens up the possibility of a finite magnetoelectric coupling. This is linked to magnetization profiles in which the spin moment smoothly winds while sweeping particular paths in coordinate space. Notably, such exotic magnetoelectric manifestations are incompatible with commensurate magnetic phases and their related IC extensions.

To be more specific regarding the type of magnetoelectric phenomena to be explored in the present work, we remind the reader that all the six new IC phases discovered here consist of a single- or double- $\boldsymbol{Q} \mathrm{MH}$ configurations. However, it is well known from other studies that a $\mathrm{MH}$ is equivalent to a SOC term in the presence of an orthogonally oriented ferromagnetic field [34,41,42]. To make the latter statement transparent, consider the MH Hamiltonian term,

$$
\begin{aligned}
\boldsymbol{M}(\boldsymbol{r}) \cdot \boldsymbol{\sigma} & =M\left[\sin (\boldsymbol{q} \cdot \boldsymbol{r}) \hat{\boldsymbol{n}}_{\perp}+\cos (\boldsymbol{q} \cdot \boldsymbol{r}) \hat{\boldsymbol{n}}\right] \cdot \boldsymbol{\sigma} \\
& =M e^{-i \boldsymbol{q} \cdot \boldsymbol{r}\left(\hat{\boldsymbol{n}} \times \hat{\boldsymbol{n}}_{\perp}\right) \cdot \boldsymbol{\sigma} / 2} \hat{\boldsymbol{n}} \cdot \boldsymbol{\sigma} e^{i \boldsymbol{q} \cdot \boldsymbol{r}\left(\hat{\boldsymbol{n}} \times \hat{\boldsymbol{n}}_{\perp}\right) \cdot \boldsymbol{\sigma} / 2}
\end{aligned}
$$

note that the unit vectors $\hat{\boldsymbol{n}}$ and $\hat{\boldsymbol{n}}_{\perp}$ comprise the complex spin vector $\hat{\boldsymbol{n}}_{\boldsymbol{q}}=\left(\hat{\boldsymbol{n}}-i \hat{\boldsymbol{n}}_{\perp}\right) / \sqrt{2}$ (up to an overall factor). For illustration purposes, let us momentarily consider free electrons under the influence of the above MH term.
Gauging away the spatially varying $\mathrm{SU}(2)$ phase above, one obtains the single electron Hamiltonian,

$$
\begin{aligned}
\frac{[\boldsymbol{p}-}{\left.\boldsymbol{q} \boldsymbol{q}\left(\hat{\boldsymbol{n}} \times \hat{\boldsymbol{n}}_{\perp}\right) \cdot \boldsymbol{\sigma} / 2\right]^{2}} & 2 m \\
= & \frac{\left(\hat{\boldsymbol{q}}_{\perp} \cdot \boldsymbol{p}\right)^{2}}{2 m}+\frac{(\hbar \boldsymbol{q})^{2}}{8 m}+\frac{(\hat{\boldsymbol{q}} \cdot \boldsymbol{p})^{2}}{2 m} \\
& -\frac{1}{2} \alpha_{q} \hat{\boldsymbol{q}} \cdot \boldsymbol{p}\left(\hat{\boldsymbol{n}} \times \hat{\boldsymbol{n}}_{\perp}\right) \cdot \boldsymbol{\sigma}+M \hat{\boldsymbol{n}} \cdot \boldsymbol{\sigma},
\end{aligned}
$$

where $\alpha_{q}=\hbar|\boldsymbol{q}| / m$ defines the strength of the generated SOC, while we introduced the unit vector $\hat{\boldsymbol{q}}=\boldsymbol{q} /|\boldsymbol{q}|$. The presence of inversion-breaking SOC mediates a magnetoelectric coupling and can lead to a number of so-called direct and inverse spin galvanic phenomena [37]. Here we focus on the generation of a homogeneous magnetization when an electric charge current (I) flows through the system or an electric field $(\mathcal{E})$ is applied. Both situations can be described by coupling the electronic system to a spatially homogeneous but generally time-dependent vector potential $\boldsymbol{A}(t)$, that in the Coulomb gauge yields the electric field $\mathcal{E}(t)=-\partial_{t} \boldsymbol{A}(t)$. Instead, if $\boldsymbol{A}$ is time independent, it corresponds to a current flow bias, i.e., $I \propto A$.

It is straightforward to infer that a vector potential minimally coupled to the momentum, i.e., $\boldsymbol{p} \rightarrow \boldsymbol{p}+e \boldsymbol{A}(t)$, will generate a time-dependent but spatially homogeneous magnetization of the form

$$
\boldsymbol{M}_{\mathbf{0}}(t) \propto\left(\hat{\boldsymbol{n}} \times \hat{\boldsymbol{n}}_{\perp}\right) \hat{\boldsymbol{q}} \cdot \boldsymbol{A}(t) \propto\left(i \hat{\boldsymbol{n}}_{\boldsymbol{q}} \times \hat{\boldsymbol{n}}_{\boldsymbol{q}}^{*}\right) \hat{\boldsymbol{q}} \cdot \boldsymbol{A}(t) .
$$

The derivation of the required magnetoelectric coupling leading to the above relation is presented in Appendix C.

Interestingly, via magnetization measurements in the zero-external magnetic field limit, one can arrest the magnetoelectrically generated ferromagnetic moment, as long as the current or electric field is orientated along the MH's spatial modulation direction $\hat{\boldsymbol{q}}$. Moreover, the spin orientation of the induced magnetization can provide information regarding the MH's winding plane, since the former is given by $i \hat{\boldsymbol{n}}_{\boldsymbol{q}} \times \hat{\boldsymbol{n}}_{\boldsymbol{q}}^{*}$. To further demystify the nature of such vectors, one observes that they can be linked to the magnetic order parameter's winding in coordinate space along the $\boldsymbol{q}$ direction, i.e.,

$$
\boldsymbol{w}_{\boldsymbol{q}} \propto \int_{\mathrm{UC}} d \boldsymbol{r} \boldsymbol{M}(\boldsymbol{r}) \times\left[\hat{\boldsymbol{q}} \cdot \frac{\partial \boldsymbol{M}(\boldsymbol{r})}{\partial \boldsymbol{r}}\right] \propto i \hat{\boldsymbol{n}}_{\boldsymbol{q}} \times \hat{\boldsymbol{n}}_{\boldsymbol{q}}^{*},
$$

with UC denoting the magnetic unit cell and $\boldsymbol{w}_{q}$ corresponding to a vector normal to the winding plane defined by the helix. 
Based on the above analysis, we find that magnetoelectric phenomena become possible in the IC magnetic phases discussed here when either one of the quantities, $i \hat{\boldsymbol{n}}_{1} \times \hat{\boldsymbol{n}}_{1}^{*}$ or $i \hat{\boldsymbol{n}}_{2} \times \hat{\boldsymbol{n}}_{2}^{*}$, becomes nonzero. In the former (latter) case, the induced SOC involves the electron's momentum along the $\boldsymbol{Q}_{1}\left(\boldsymbol{Q}_{2}\right)$ direction. For the IC magnetic phases and respective order parameters under investigation, we observe that only the six new phases lead to magnetoelectric phenomena, as summarized in Table I.

In fact, the $\mathrm{SWC}_{4,2}$ phases consist of two noncoplanar MHs and can give rise to such phenomena for an arbitrary orientation of $\boldsymbol{I}$ or $\mathcal{E}$, since both external products are nonzero and nonparallel. In the present case, Eq. (18) and Appendix $\mathrm{C}$ imply that the magnetization retains contributions from both $\mathrm{MHs}$, and one thus obtains

$\boldsymbol{M}_{\mathbf{0}}(t) \propto\left(i \hat{\boldsymbol{n}}_{1} \times \hat{\boldsymbol{n}}_{1}^{*}\right) \boldsymbol{Q}_{1} \cdot \boldsymbol{A}(t)+\left(i \hat{\boldsymbol{n}}_{2} \times \hat{\boldsymbol{n}}_{2}^{*}\right) \boldsymbol{Q}_{2} \cdot \boldsymbol{A}(t)$.

In contrast, the remaining magnetic phases induce SOC only along one direction, and thus are not capable of exhibiting magnetoelectric phenomena when the external field is not properly aligned. The reason is that three of those consist only of a single $\mathrm{MH}$ that essentially singles out the direction for which such effects become accessible. Interestingly, the same holds for the DPMH phase due to the coplanarity of the underlying MHs. Therefore, we start from Eq. (20) and set $\hat{\boldsymbol{n}}_{1}=\hat{\boldsymbol{n}}_{2}$ that dictates the DPMH phase. The latter simply yields

$$
\boldsymbol{M}_{\mathbf{0}}(t) \propto\left(i \hat{\boldsymbol{n}}_{1} \times \hat{\boldsymbol{n}}_{1}^{*}\right)\left(\boldsymbol{Q}_{1}+\boldsymbol{Q}_{2}\right) \cdot \boldsymbol{A}(t),
$$

revealing that magnetoelectric phenomena are not accessible when $\mathcal{E} \|\left(\boldsymbol{Q}_{1}-\boldsymbol{Q}_{2}\right)$.

To this end, let us remark that while we focused here on the generation of a ferromagnetic moment when an electric field is applied or a current flow is induced, reciprocal effects are also possible, by virtue of the magnetoelectric coupling (see Appendix C). This can be particularly useful for the case of the itinerant magnets discussed here, which are expected to be good conductors and, thus, screen the externally applied fields. Therefore, depending on the case, it may appear experimentally more feasible to observe a reciprocal magnetoelectric effect, such as inducing a current by an externally imposed timedependent homogeneous magnetization or by virtue of the Zeeman coupling to an applied magnetic field. Both need to have an appropriate orientation for the magnetoelectric effects to take place. Based on Appendix C, a field of frequency $\omega, \boldsymbol{M}_{0}(\omega)$, generates an electric current $\boldsymbol{J}(\omega)$, which reads

$$
\boldsymbol{Q}_{s} \cdot \boldsymbol{J}(\omega) \propto\left(i \hat{\boldsymbol{n}}_{s} \times \hat{\boldsymbol{n}}_{s}^{*}\right) \cdot \boldsymbol{M}_{\boldsymbol{0}}(\omega) .
$$

In the above, we projected the current along the directions of the magnetic wave vectors $\boldsymbol{Q}_{s}(s=1,2)$.

\section{Zeeman-field-induced spin-Skyrmion crystals}

As discussed earlier, the SWC phases are special as, in contrast to the remaining set of magnetic profiles examined here, they are characterized by isolated $\pi$-Berry flux sources. This feature is essentially responsible for the observed distinct behavior of these phases regarding the occurrence of magnetoelectric phenomena independent of the orientation of the electric field. When the $\Theta$ symmetry is broken, one obtains $|\boldsymbol{M}(\boldsymbol{r})| \neq 0 \forall \boldsymbol{r}$ and the SWC phases allow for the engineering of more exotic magnetic textures, the so-called spin-Skyrmion crystal phases. The latter are characterized by a magnetic Skyrmion charge retrieved via the Chern number of the magnetic unit vector $\hat{\boldsymbol{n}}(\boldsymbol{r})=$ $\boldsymbol{M}(\boldsymbol{r}) /|\boldsymbol{M}(\boldsymbol{r})|[73]:$

$$
\begin{aligned}
\mathcal{C} & =\frac{1}{4 \pi} \int_{\mathrm{UC}} d \boldsymbol{r} \hat{\boldsymbol{n}}(\boldsymbol{r}) \cdot\left[\frac{\partial \hat{\boldsymbol{n}}(\boldsymbol{r})}{\partial x} \times \frac{\partial \hat{\boldsymbol{n}}(\boldsymbol{r})}{\partial y}\right] \\
& \equiv \frac{1}{2 \pi} \int_{\mathrm{UC}} d \boldsymbol{r} \Omega_{x y}(\boldsymbol{r})
\end{aligned}
$$

with UC denoting the magnetic unit cell, determined by the ordering wave vectors $\boldsymbol{Q}_{1,2}$. In fact, the $\mathrm{SWC}_{4}$ phase constitutes a critical magnetic phase, and lies on the border separating two spin-Skyrmion crystal phases of different Skyrmion charge.

To engineer a spin-Skyrmion crystal from the SWC phases it is required to violate the $\Theta$ symmetry. While it is expected that the incommensurability will violate it in the actual material, the outcome is difficult to predict and, thus, a control knob to induce a nonzero Skyrmion charge is certainly desirable. One way to achieve the latter is to externally apply a homogeneous Zeeman field, that is even under translations and odd under $\mathcal{T}$. Via the application of the Zeeman field we are additionally in a position to control the sign of $\mathcal{C}$ [55], which also specifies the orientation of the out-of-plane orbital angular momentum in the system [67]. Note that while an arbitrarily weak Zeeman field violates the $\Theta$ symmetry, $\mathcal{C}$ is nonzero only within a particular window of the Zeeman energy values, $E_{\text {Zeeman }}$.

One finds that, given the expressions of Eqs. (12) and (14), the SWC phases become Skyrmionic by applying a Zeeman field $\mathcal{B}=\mathcal{B} \hat{z}$. The straightforward calculation of $\mathcal{C}$ yields that $|\mathcal{C}|=1$ for both phases. In the case of the $\mathrm{SWC}_{4}$ phase, the Skyrmion charge is nonzero for $\left|E_{\text {Zeeman }}\right|<2 \sqrt{2} M|\sin \lambda|$, i.e., even for an infinitesimal applied field. In contrast, we find that a threshold field is required to induce a nonzero Skyrmion charge for the $\mathrm{SWC}_{2}$ phase, which is proportional to the degree of nematicity of the magnetic order parameter. Specifically, the magnetic profile is Skyrmionic for $\sqrt{2} M|A-B|<\left|E_{\text {Zeeman }}\right|<\sqrt{2} M(A+B)$, with $A=\cos \eta$ and $B=\sqrt{2} \sin \eta|\cos \lambda|$. Finally, note that the presence of SOC will pin the magnetic moment direction, assumed free 
in Eqs. (12) and (14), and thus constrain the actual orientation of the required Zeeman field. See also Sec. VIC.

\section{INCOMMENSURATE MAGNETIC PHASES IN THE IRON-BASED SUPERCONDUCTORS}

Having obtained the possible leading magnetic instabilities and their distinctive characteristics for the general class of tetragonal itinerant systems described phenomenologically via the Landau free energy of Eq. (1), we may now proceed by exploring the possibility of such phases appearing in FeSCs. To accomplish this task we extract the coefficients of the free-energy functional from two representative multiorbital microscopic models. These are supplemented with standard Hubbard-Hund interactions that can drive the system to a magnetic instability. Below, we present the details of this analysis.

\section{A. Microscopic model}

We adopt a microscopic Hamiltonian, $\mathcal{H}=\mathcal{H}_{0}+\mathcal{H}_{\text {int }}$, with

$$
\mathcal{H}_{0}=\sum_{\boldsymbol{k}} \sum_{\substack{a b \\ \sigma}}\left[\epsilon_{a b}(\boldsymbol{k})-\mu \delta_{a b}\right] c_{\boldsymbol{k} a \sigma}^{\dagger} c_{\boldsymbol{k} b \sigma},
$$

provided by tight-binding fits to DFT calculations. Here we consider two five-orbital band structures, adopted from Refs. [58,59], appropriate for $\mathrm{BaFe}_{2} \mathrm{As}_{2}$ and $\mathrm{LaFeAsO}$, respectively. The orbital basis is $\left(x z, y z, x y, x^{2}-y^{2}, z^{2}\right)$. Although, the $\mathrm{BaFe}_{2} \mathrm{As}_{2}$ band structure is three dimensional, here we restrict to the $k_{z}=0$ plane. The band structures are supplemented by $\mathcal{H}_{\text {int }}$ consisting of HubbardHund interactions [77,78]:

$$
\begin{aligned}
\mathcal{H}_{\mathrm{int}}= & \frac{U}{\mathcal{N}} \sum_{\boldsymbol{q}} \sum_{a} n_{\boldsymbol{q} a \uparrow} n_{-q a \downarrow}+\frac{U^{\prime}}{\mathcal{N}} \sum_{\boldsymbol{q}} \sum_{\substack{a<b \\
\sigma \sigma^{\prime}}} n_{\boldsymbol{q} a \sigma} n_{-\boldsymbol{q} b \sigma^{\prime}}+\frac{J}{2 \mathcal{N}} \sum_{\substack { \boldsymbol{k} \boldsymbol{k}^{\prime} \boldsymbol{q} \\
\begin{subarray}{c}{a \neq b \\
\sigma \sigma^{\prime}{ \boldsymbol { k } \boldsymbol { k } ^ { \prime } \boldsymbol { q } \\
\begin{subarray} { c } { a \neq b \\
\sigma \sigma ^ { \prime } } }\end{subarray}} c_{\boldsymbol{k}+\boldsymbol{q} a \sigma}^{\dagger} c_{\boldsymbol{k} b \sigma} c_{\boldsymbol{k}^{\prime}-\boldsymbol{q} b \sigma^{\prime}}^{\dagger} c_{\boldsymbol{k}^{\prime} a \sigma^{\prime}} \\
& +\frac{J^{\prime}}{2 \mathcal{N}} \sum_{\boldsymbol{k} \boldsymbol{k}^{\prime} \boldsymbol{q}} \sum_{\substack{a \neq b \\
\sigma}} c_{\boldsymbol{k}+\boldsymbol{q} a \sigma}^{\dagger} c_{\boldsymbol{k}^{\prime}-\boldsymbol{q} a \bar{\sigma}}^{\dagger} c_{\boldsymbol{k}^{\prime} b \bar{\sigma}} c_{\boldsymbol{k} b \sigma}
\end{aligned}
$$

where, by imposing $\mathrm{SO}(3)$ spin-rotational invariance on the sum of the interaction terms and further assuming orbitalindependent interactions, we obtain $U^{\prime}=U-2 J$ and $J=J^{\prime}$. In the above expressions, $c_{k a \sigma}^{\dagger}\left(c_{k a \sigma}\right)$ creates (annihilates) an electron in orbital $a$ with momentum $\boldsymbol{k}$ and spin $\sigma . \mathcal{N}$ denotes the number of momentum states.
The above interaction terms are treated at the mean-field level via a Hubbard-Stratonovich decoupling in the magnetic channel, yielding an effective action for the magnetic order parameters. Expanding to quartic order yields an extension of the magnetic free energy in Eq. (1) to include the orbital content of the magnetic order parameters:

$$
\begin{aligned}
F= & \sum_{q} \sum_{a b c d}\left[\left(U^{-1}\right)^{a b c d}-\chi_{0}^{a b c d}(\boldsymbol{q})\right] \boldsymbol{M}^{a b}(\boldsymbol{q}) \boldsymbol{M}^{c d}(-\boldsymbol{q})+\sum_{\substack{a b c d \\
e f g h}} \tilde{\beta}_{1}^{a b c d e f g h}\left(\boldsymbol{M}_{1}^{a b} \cdot \boldsymbol{M}_{1}^{c d *}\right)\left(\boldsymbol{M}_{1}^{e f} \cdot \boldsymbol{M}_{1}^{g h *}\right) \\
& +\sum_{\substack{a b c d \\
e f g h}} \tilde{\beta}_{2}^{a b c d e f g h}\left(\boldsymbol{M}_{2}^{a b} \cdot \boldsymbol{M}_{2}^{c d *}\right)\left(\boldsymbol{M}_{2}^{e f} \cdot \boldsymbol{M}_{2}^{g h *}\right)+\frac{1}{2} \sum_{\substack{a b c d \\
e f g h}}\left(\beta_{1}-\tilde{\beta}_{1}\right)^{a b c d e f g h}\left(\boldsymbol{M}_{1}^{a b} \cdot \boldsymbol{M}_{1}^{c d}\right)\left(\boldsymbol{M}_{1}^{e f *} \cdot \boldsymbol{M}_{1}^{g h *}\right) \\
& +\frac{1}{2} \sum_{\substack{a b c d \\
\text { efgh }}}\left(\beta_{2}-\tilde{\beta}_{2}\right)^{a b c d e f g h}\left(\boldsymbol{M}_{2}^{a b} \cdot \boldsymbol{M}_{2}^{c d}\right)\left(\boldsymbol{M}_{2}^{e f *} \cdot \boldsymbol{M}_{2}^{g h *}\right)+\sum_{\substack{a b c d \\
e f g h}} g^{a b c d e f g h}\left(\boldsymbol{M}_{1}^{a b} \cdot \boldsymbol{M}_{1}^{c d *}\right)\left(\boldsymbol{M}_{2}^{e f} \cdot \boldsymbol{M}_{2}^{g h *}\right) \\
& +\frac{1}{2} \sum_{\substack{a b c d \\
\text { efgh }}} \tilde{g}_{1}^{a b c d e f g h}\left(\boldsymbol{M}_{1}^{a b} \cdot \boldsymbol{M}_{2}^{c d}\right)\left(\boldsymbol{M}_{1}^{e f *} \cdot \boldsymbol{M}_{2}^{g h *}\right)+\frac{1}{2} \sum_{\substack{a b c d \\
\text { efgh }}} \tilde{g}_{2}^{a b c d e f g h}\left(\boldsymbol{M}_{1}^{a b} \cdot \boldsymbol{M}_{2}^{c d *}\right)\left(\boldsymbol{M}_{1}^{e f *} \cdot \boldsymbol{M}_{2}^{g h}\right) .
\end{aligned}
$$

We note that the coefficients of the free energy are tensors in orbital space and are entirely determined by the microscopic band structure. The expressions for these coefficients are given in Appendix D. Here we retained the $\boldsymbol{q}$ dependence of the magnetic order at quadratic level to identify the transition from commensurate to IC magnetism. In contrast, the quartic order terms are calculated for fixed $\boldsymbol{q}$, i.e., $\boldsymbol{Q}_{1,2}$, which are set by the quadratic term.
To determine the magnetic transition temperature, we follow the procedure described in Ref. [11] and consider the generalized eigenvalue problem,

$$
\left[\mathcal{D}_{\mathrm{mag}}^{-1}(\boldsymbol{q})\right]^{a b c d} v_{c d}(\boldsymbol{q})=\lambda(\boldsymbol{q}) v_{a b}(\boldsymbol{q}),
$$

where 
$\mathrm{BaFe}_{2} \mathrm{As}_{2}$

(a)

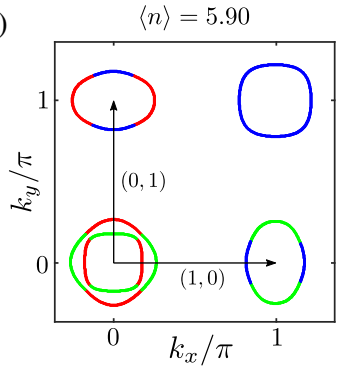

(c)

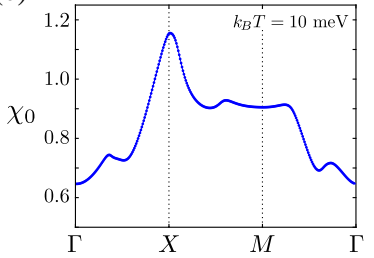

(e)

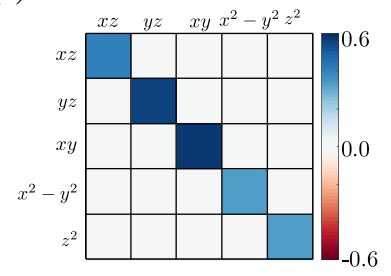

(b)



(d)

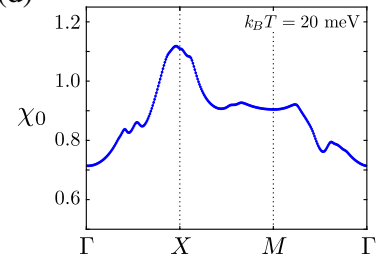

(f)

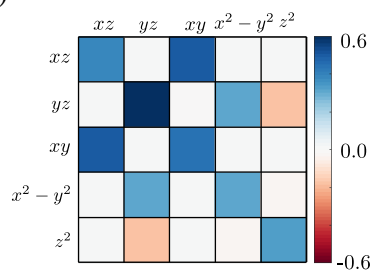

(g)

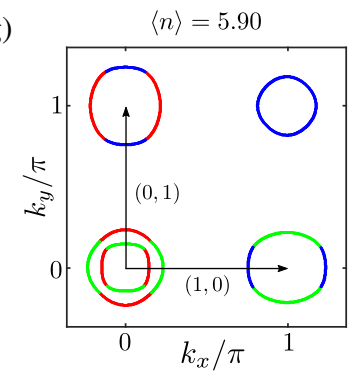

(i)



(k)

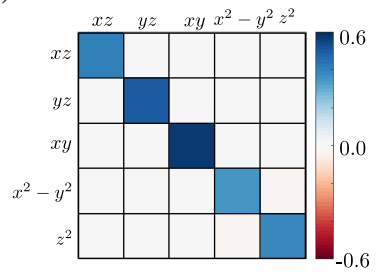

$\mathrm{LaFeAsO}$

(h)

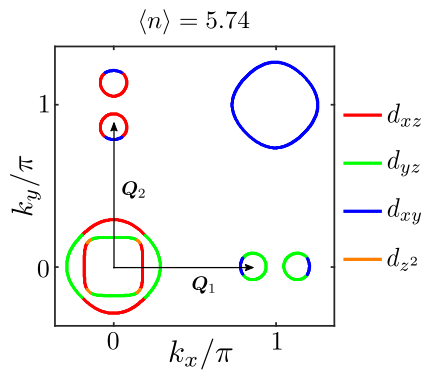

(j)

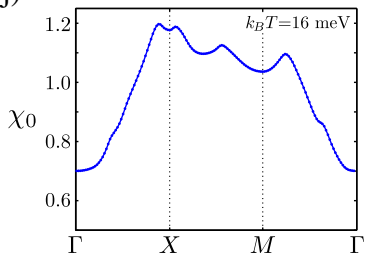

(l)



FIG. 4. Fermi surfaces, bare physical susceptibilities $\sum_{a b} \chi_{0}^{a a b b}(\boldsymbol{q})$, and orbital content at the magnetic transitions for (a)-(f) BaFe $\mathrm{As}_{2}$ and (g)-(1) LaFeAsO. The Fermi surface depicted in (a) for $\langle n\rangle=5.90$ results in a commensurate magnetic order while the Fermi surface in (b) with $\langle n\rangle=5.76$ leads to incommensurate magnetic order. We note that both these refer to the $k_{z}=0$ slice of the $\mathrm{BaFe}_{2} \mathrm{As}_{2}$ band structure of Ref. [58]. In the commensurate case, the susceptibility is depicted in (c), clearly exhibiting a peak at the $X$ point $(\pi, 0)$. In contrast, in the incommensurate case (d), the peak is displaced from $(\pi, 0)$ to $(\pi-\delta, 0)$. For the case shown in (d), $\langle n\rangle=5.76$ and $\delta=\pi / 50$. Incommensurability in the $\mathrm{BaFe}_{2} \mathrm{As}_{2}$ band structure onsets at $\langle n\rangle=5.80$, for which $\delta=\pi / 200$. Furthermore, we note that the path between $\Gamma$ and $X$ is dominated by a single peak, and while subleading peaks are present, these are clearly much less pronounced. This allows us to neglect higher harmonics of $\boldsymbol{Q}=(Q, 0) /(0, Q)$. The orbital content of the magnetic order parameter at the transition is shown in (e) and (f) for the commensurate and incommensurate cases, respectively. In the commensurate case the contributions are purely real. The additional terms appearing as magnetism becomes incommensurate are imaginary, and for purposes of the presentation have been multiplied by a factor of 10 . Hence, in the IC case, $\boldsymbol{M}\left(\boldsymbol{Q}_{1,2}\right) \neq \boldsymbol{M}\left(-\boldsymbol{Q}_{1,2}\right)$, as this would result in a change of sign of the imaginary parts. In (g) we show the Fermi surface for the LaFeAsO band structure with $\langle n\rangle=5.90$. The commensurate-toincommensurate transition is brought on by a change in Fermi surface topology, as seen in (h). As in the case of $\mathrm{BaFe}_{2} \mathrm{As}_{2}$, the bare susceptibility depicted in (j) shows a clear peak at $(\pi-\delta, 0)$, although in this case $\delta \approx \pi / 10$ even close to the transition to incommensurate magnetism. The orbital content of the magnetic order parameter depicted in (k) and (1) is very similar to the $\mathrm{BaFe}_{2} \mathrm{As}_{2}$ band structure, although the $x y$ orbital is more dominant in the case of LaFeAsO.

$$
\left[\mathcal{D}_{\text {mag }}^{-1}(\boldsymbol{q})\right]^{a b c d}=\left(U^{-1}\right)^{a b c d}-\chi_{0}^{a b c d}(\boldsymbol{q})
$$

denotes the (inverse) magnetic propagator, $\lambda(\boldsymbol{q})$ is the smallest eigenvalue, and $v_{a b}(\boldsymbol{q})$ is the associated eigenmatrix. The vanishing of $\lambda(\boldsymbol{Q})$ signals the onset of magnetic order with ordering vector $\boldsymbol{Q}$. From the vanishing of $\lambda(\boldsymbol{Q})$ we find three types of ordering vectors appearing as a function of electron filling (see insets in Fig. 5). The orbital weight of the order parameter with ordering vector $Q$ is contained in the matrix $v_{a b}(\boldsymbol{Q})$ and we write $\boldsymbol{M}_{1,2}^{a b}=\boldsymbol{M}_{1,2} v_{a b}\left(\boldsymbol{Q}_{1,2}\right)$. The quadratic coefficient provides information on the transition temperature, and the quartic coefficients are required for the determination of the leading magnetic instability. The relation between the quartic coefficients and the microscopic band structure is provided by the above Hubbard-Stratonovich decoupling, and due to the IC nature of the ordering vectors it is necessary to truncate the expressions. We accomplish this by assuming that only the lowest harmonics contribute, an assumption justified by comparing the magnitude of the peak at $\boldsymbol{Q}_{1,2}$ in the bare susceptibility with the peaks at higher integer multiples of $\boldsymbol{Q}_{1,2}$, as shown in Figs. 4(d) and 4(j), for the $\mathrm{BaFe}_{2} \mathrm{As}_{2}$ and $\mathrm{LaFeAsO}$ band structures. The eigenmatrix $v_{a b}\left(\boldsymbol{q}=\boldsymbol{Q}_{1,2}\right)$ encodes the orbital content of the magnetic order parameter at the magnetic transition [11]. Note that this object contains information solely about the magnetic order parameter's orbital structure, and not its magnitude. Below, we 
$\mathrm{BaFe}_{2} \mathrm{As}_{2}$

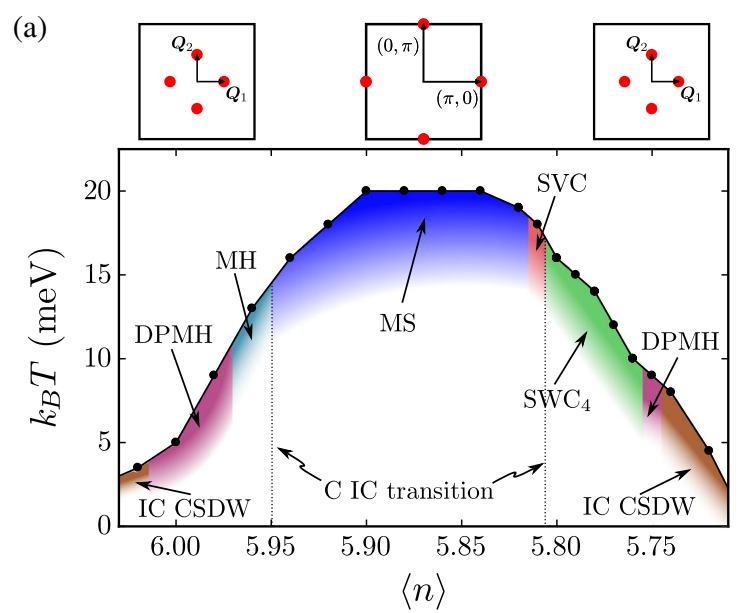

(b)

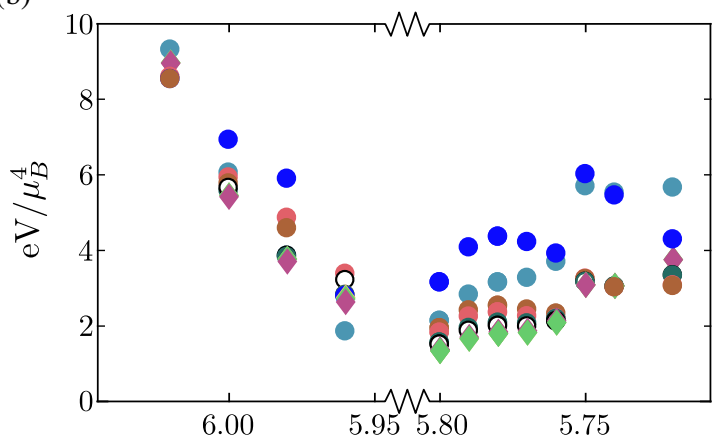

$\langle n\rangle$
LaFeAsO

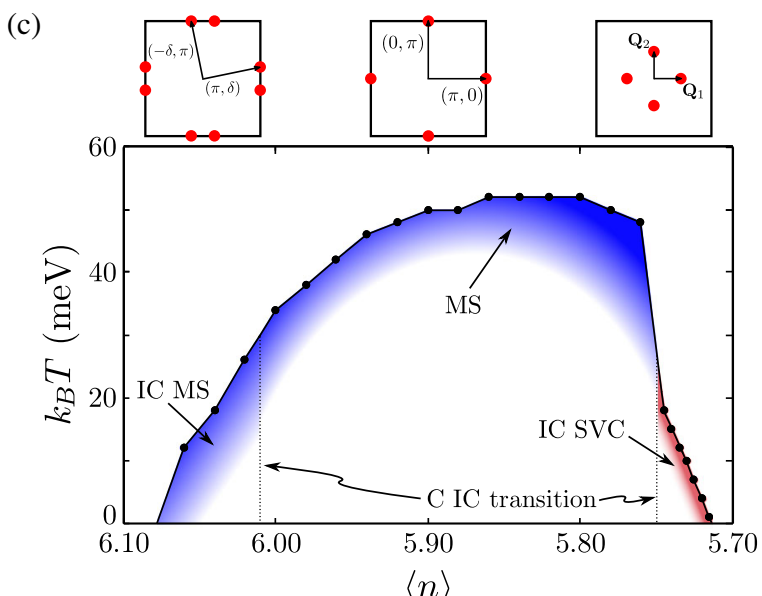

(d)

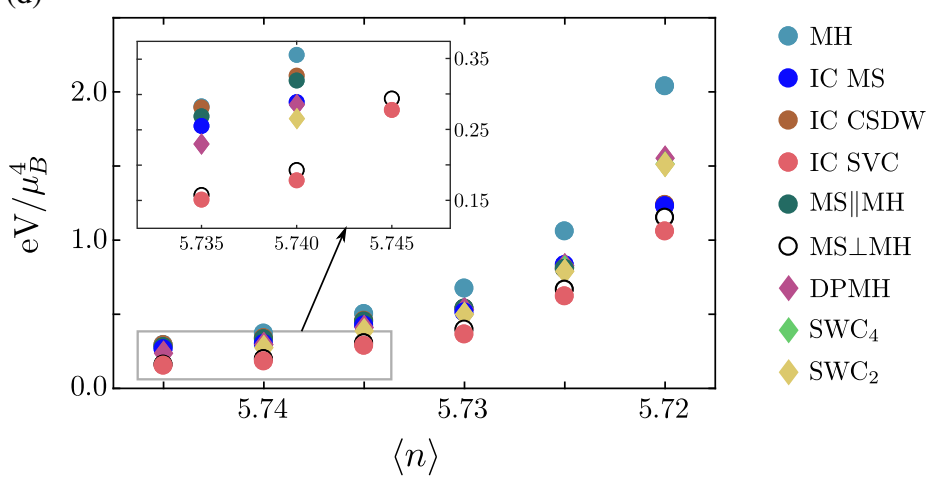

FIG. 5. Magnetic transition temperatures, leading instabilitites, and free energies as a function of the filling $\langle n\rangle$ for two representative five-orbital models corresponding to (a),(b) $\mathrm{BaFe}_{2} \mathrm{As}_{2}$ and (c),(d) $\mathrm{LaFeAsO}$. The type of incommensurability, $(\pi-\delta, 0) /(0, \pi-\delta)$ or $(\pi, \delta) /(-\delta, \pi)$, is illustrated above (a) and (c). For $\mathrm{BaFe}_{2} \mathrm{As}_{2}$ we find the usual MS phase in the majority of the commensurate region of the phase diagram; see (a). Prior to the transition to an incommensurate phase, a $C_{4} \mathrm{SVC}$ phase sets in. Incommensurate magnetism occurs for $\langle n\rangle>5.95$ and $\langle n\rangle<5.81$. In both cases $Q_{1,2}=(\pi-\delta, 0) /(0, \pi-\delta)$, with $\delta$ evolving smoothly from zero. We find the MH, DPMH, and IC-CSDW phases for $\langle n\rangle>5.95$ and the SWC 4 , DPMH, and IC-CSDW phases for $\langle n\rangle<5.81$. In (b) the free energies of the various phases in the IC region are shown, indicating the relative proximity of all the IC phases. The full expressions are provided in Appendix A. In (c), the leading instabilities for $\mathrm{LaFeAsO}$ are depicted. The electron-doped side with $\langle n\rangle>6$ exhibits IC magnetic order with ordering vector $\boldsymbol{Q}_{1,2}=(\pi, \delta) /(-\delta, \pi)$ and IC-MS order. In the commensurate region, the usual MS phase is found. Magnetism becomes incommensurate again on the hole-doped side for $\langle n\rangle<5.75$, although in this case $\boldsymbol{Q}_{1,2}=(\pi-\delta, 0) /(0, \pi-\delta)$. However, this commensurate-to-incommensurate transition occurs due to the change in topology of the Fermi surface shown in Fig. 4(h) and $\delta$ jumps from zero to a finite value, $\delta \approx \pi / 10$. The magnetic phase in the IC region is the IC-SVC phase. (d) Magnitude of $4 F^{(4)} / M^{4}$ for the different IC magnetic phases for $U=0.95 \mathrm{eV}$ and $J=U / 4$. As the magnetic order becomes incommensurate, the IC-SVC phase is favored. The inset shows an enlargement of the lower left-hand corner.

employ the matrices $v_{a b}\left(\boldsymbol{Q}_{1,2}\right)$ to project Eq. (26) onto the leading instability. Thus, we obtain the free energy of Eq. (1) with the coefficients obtained from the microscopic models; see Eqs. (D2)-(D7) of Appendix D.

The incommensurability evident in Figs. 4(d) and 4(j) is caused by a change in the Fermi surface nesting properties induced by sufficient hole or electron doping. In Figs. 4(a) and $4(\mathrm{~g})$, we show the Fermi surfaces for both the $\mathrm{BaFe}_{2} \mathrm{As}_{2}$ and $\mathrm{LaFeAsO}$ band structures in the paramagnetic phase, at a doping leading to commensurate magnetic order, i.e., $\langle n\rangle=5.90$. We also show the Fermi surfaces for systems with substantial hole doping, leading to IC magnetic orders; see Figs. 4(b) and 4(h). Note that the doping necessary to drive the IC transition is substantial, i.e., the commensurate order is preserved even under moderate doping, consistent with experimental observations. In the case of $\mathrm{BaFe}_{2} \mathrm{As}_{2}$ the Fermi surface is not drastically modified as the magnetic transition becomes incommensurate; see Figs. 4(a) and 4(b). For $\mathrm{LaFeAsO}$, on the other hand, the IC transition occurs as a result of the change in topology of the electron pockets at $X$ and $Y$; see Fig. 4(h). This is reflected in the suppression of the magnetic transition temperature in Fig. 5(c). In Fig. 4 we also present the 
orbital content obtained from $v_{a b}\left(\boldsymbol{Q}_{1,2}\right)$ for both commensurate [(Figs. 4(e) and 4(k)] and IC magnetism [Figs. 4(f) and 4(1)] for the two band structures. We observe that, as the ordering vector becomes IC, the orbitally resolved magnetic order parameter acquires imaginary components, an indication that indeed $\boldsymbol{M}\left(\boldsymbol{Q}_{1,2}\right) \neq \boldsymbol{M}\left(-\boldsymbol{Q}_{1,2}\right)$, as expected.

\section{B. Magnetic phase diagram}

We determine the location of the magnetic transition and the respective leading instability as a function of the electron filling $\langle n\rangle$, for $U=0.95 \mathrm{eV}$ and $J=U / 4$, for the two band structures described above. The results are depicted in Fig. 5. In both cases we find a large region of commensurate magnetism with $\boldsymbol{Q}=(\pi, 0) /(0, \pi)$, around a filling of $\langle n\rangle \approx 5.90$ with IC phases appearing upon either hole or electron doping, as the Fermi surface nesting properties are sufficiently modified. As expected based on the picture of itinerant magnetism, the magnetic order becomes IC as the Fermi surface is substantially deformed by the addition or removal of carriers. For the $\mathrm{BaFe}_{2} \mathrm{As}_{2}$ band structure, both electron and hole doping leads to IC phases with $\boldsymbol{Q}_{1,2}=(\pi-\delta, 0) /(0, \pi-\delta)$, with $\delta$ increasing smoothly from zero. The transition temperature broadly follows the expected behavior [25,26], exhibiting an inflection point at the commensurate-to-incommensurate transition; see Fig. 5(a). On the electron-doped side, we find two $C_{2}$-symmetric phases, in addition to the $C_{4}$-symmetric IC-CSDW phase. These are the $\mathrm{MH}$ and DPMH phases. On the hole-doped side we find a transition to a $C_{4} \mathrm{SVC}$ phase prior to the commensurateto-incommensurate transition. As magnetism becomes incommensurate, the $\mathrm{SWC}_{4}$ phase becomes favored, followed by the DPMH and the IC-CSDW phases.

In contrast, for the $\mathrm{LaFeAsO}$ band structure, electron doping yields a region with $\boldsymbol{Q}_{1,2}=(\pi, \delta) /(-\delta, \pi)$ and IC magnetic stripe order, as seen in Fig. 5(c). In this case, $\delta$ also smoothly increases from zero. Substantial hole doping (i.e., $\langle n\rangle \approx 5.75$ ) yields the IC magnetic wave vectors $\boldsymbol{Q}_{1,2}=(\pi-\delta, 0) /(0, \pi-\delta)$, similar to the case of the $\mathrm{BaFe}_{2} \mathrm{As}_{2}$ band structure. However, in this case $\delta$ exhibits a jump from zero to a finite value, associated with the change in topology of the Fermi surface shown in Fig. 4(h). This also leads to a suppression of the magnetic transition temperature, as seen in Fig. 5(c). The commensurate magnetic stripe phase occupying a large part of the phase diagram is succeeded by the IC-SVC phase, which persists until the end of the magnetic dome.

In Figs. 5(b) and 5(d) we show the free energy of the various phases in the IC region. More specifically, we plot the value of $4 F^{(4)} / M^{4}$, where $F^{(4)}$ is the quartic part of the free energy in Eq. (1), as discussed in Appendix A. Note that this number does not take into account the effect of the increasing magnitude of the magnetic order parameter. Thus, it is valid only in the vicinity of the transition. For the
$\mathrm{BaFe}_{2} \mathrm{As}_{2}$ band structure, many phases appear in close proximity, leading to a number of different leading instabilities, as seen in Fig. 5(a). In the case of LaFeAsO [Fig. 5(d)], the IC-SVC phase is favored throughout the IC region, although the remaining phases appear close in energy.

The small free-energy separations observed in Figs. 5(b) and 5(d) suggest that the phases stabilized are sensitive to specific details of the microscopic models. To highlight this sensitivity, we consider the effect of small changes to the interaction parameters, inspired by recent proposals of orbital selectivity $[62,63,79-81]$. Hence, we allow the interactions in Eq. (25) to exhibit orbital dependence. Despite the fact that such an orbital dependence is typically ignored, it is well known to be present in realistic models, and recent experimental and theoretical works $[62,63,79$ 81] have reemphasized its important role. Nevertheless, the consideration of such an orbital dependence introduces a large parameter space, and an exhaustive treatment is beyond the scope of the current work. Instead, we focus on varying the interactions of the $x y$ orbital. This is expected to be the most relevant for the present analysis since it is associated with the largest orbital weight, as seen in Fig. 4. To assess the impact of such orbital dependence, we vary the $x y$ component of $\left(U^{-1}\right)^{a b c d}$ in Eq. (28) and repeat the above analysis for the $\mathrm{LaFeAsO}$ band. We take
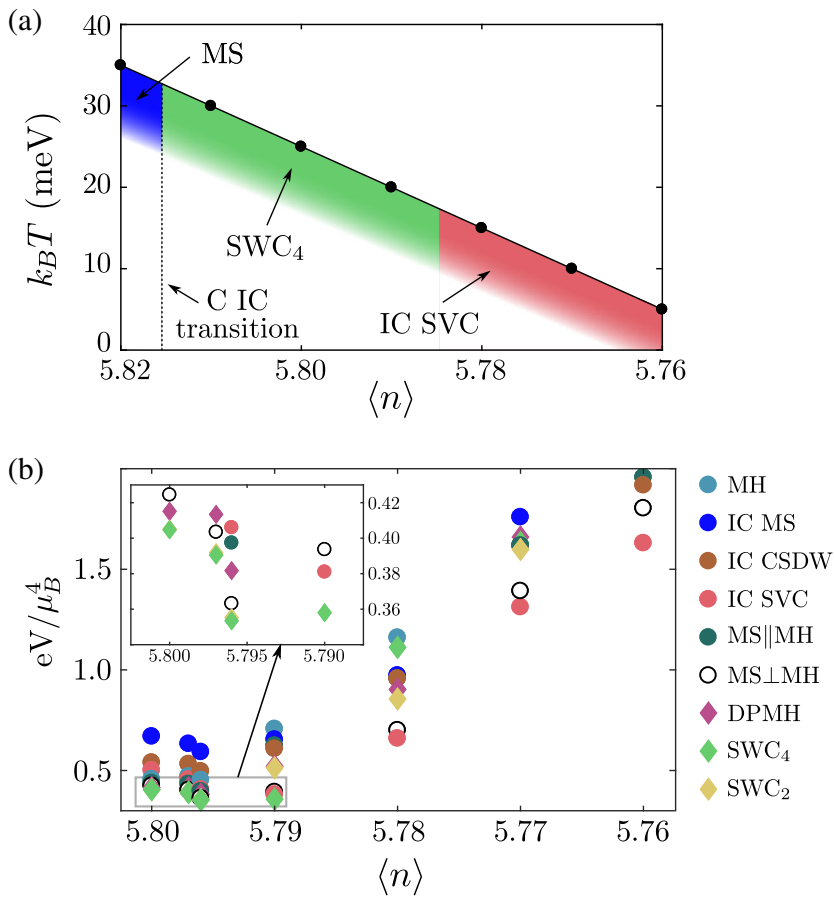

FIG. 6. (a) Phase diagram for the LaFeAsO band structure of Ref. [59] with $U=0.86 \mathrm{eV}, J=U / 4$, and $U_{x y}=1.1 U$; i.e., the $x y$ orbital is subject to a stronger interaction. The slight change in interaction parameters has changed the ground state in a region of the phase diagram, from to IC SVC to $\mathrm{SWC}_{4}$. The degree of incommensurability $\delta$ is the same as the one shown in Fig. 5(c). (b) Value of $4 F^{(4)} / M^{4}$ for the case with orbital-dependent interactions. The $\mathrm{SWC}_{4}$ phase is favored close to $\langle n\rangle \approx 5.80$. 
$U=0.86 \mathrm{eV}, \quad U_{x y}=1.1 U \approx 0.95 \mathrm{eV}, \quad$ and $\quad J=U / 4$. Unsurprisingly, we find a magnetic dome with a slightly reduced transition temperature compared to the one shown in Fig. 5(c). A commensurate-to-IC transition occurs around $\langle n\rangle \approx 5.805$ and, interestingly, the lowest-energy phase in the immediate vicinity of this transition is the $\mathrm{SWC}_{4}$, as seen in Fig. 6(a).

Based on Figs. 5 and 6, we verify that the magnetic phase diagram is sensitive to both the band structure and the interaction parameters. The appearance of the $\mathrm{SWC}_{4}$ phase implies that the FeSCs are indeed potential candidates for textured magnetic phases and, given the reported coexistence of magnetism and superconductivity [13,24,29-33], opens perspectives for realizing intrinsic topological superconductivity [82].

\section{Effects of spin-orbit coupling}

So far we have investigated the accessible magnetic phases via the free energy of Eq. (1), which neglects the effects of SOC. Here we extend our approach in order to include the effects of a SOC respecting the symmetries of the system [83]. At leading order, the free energy is modified by the addition of the term

$$
\begin{aligned}
\delta F= & \alpha_{1}\left(\left|M_{1, x}\right|^{2}+\left|M_{2, y}\right|^{2}\right)+\alpha_{2}\left(\left|M_{2, x}\right|^{2}+\left|M_{1, y}\right|^{2}\right) \\
& +\alpha_{3}\left(\left|M_{1, z}\right|^{2}+\left|M_{2, z}\right|^{2}\right),
\end{aligned}
$$

where the $\alpha$ coefficients can be determined from a microscopic model including an atomic $\boldsymbol{L} \cdot \boldsymbol{S}$ coupling $[10,84]$. Note, however, that a complete picture regarding SOC in FeSCs requires the consideration of a ten-band model $[85,86]$, taking into account the two inequivalent $\mathrm{Fe}$ sites of the FeAs layers. Lastly, note that experimental evidence points to $\alpha_{1}$ being the smallest for underdoped compounds, as these display in-plane magnetic order. For systems approaching optimal hole doping, the magnetic moments reorient out of plane [1,14], indicating that $\alpha_{3}$ is the smallest [87-90].

As the above term adds a quadratic contribution to the free energy, it plays an important role in selecting the magnetic order at the leading instability. Moreover, the additional terms modify the magnetic transition temperature $T_{\mathrm{mag}}$, obtained for vanishing SOC. Hence, $T_{\mathrm{mag}}$ is now replaced by $T_{\mathrm{mag}}^{\mathrm{SOC}}$. The normalized difference $\delta T_{\mathrm{mag}}=$ $\left(T_{\mathrm{mag}}^{\mathrm{SOC}}-T_{\mathrm{mag}}\right) / T_{\mathrm{mag}}$ can be either positive or negative and yields a quantitative measure of the SOC strength. Here we assume that the SOC is weak [91], i.e., $\left|\delta T_{\mathrm{mag}}\right| \ll 1$, a realistic assumption for FeSCs [92]. The presence of SOC also implies that only certain components of the magnetic order parameter can condense below the critical temperature $T_{\mathrm{mag}}^{\mathrm{SOC}}$, and these are selected by the smallest of the $\alpha$ coefficients. At first sight this would imply that the energetics shown in Figs. 5 and 6, obtained without SOC, can be drastically altered when $\delta F$ is added. However, as we discuss below, the results obtained with SOC remain relevant by virtue of secondary transitions.

As seen from Eq. (29), if either $\alpha_{1}$ or $\alpha_{2}$ is the smallest, the magnetic moments align in the FeAs plane, either parallel or perpendicular to the ordering vector. If instead $\alpha_{3}$ is the smallest, the moments point out of plane. Thus, only a subset of the nine IC magnetic phases found earlier are compatible with the magnetic moment directions fixed by the SOC. This subset consists of the IC generalizations of the three commensurate phases: IC- $\{$ MS, CSDW, SVC $\}$. The possible emergence of the IC-MS phase is completely unaffected by the presence of SOC, as can be inferred from Eq. (29). On the other hand, the appearance of the IC SVC (IC CSDW) is hindered if $\alpha_{3}\left(\alpha_{1}\right.$ or $\left.\alpha_{2}\right)$ is the smallest coefficient. In such situations the IC SVC or IC CDSW will only appear as the result of secondary transitions. Similarly, this is always the case for the six textured magnetic phases which are generally disfavored when SOC is present. The appearance of these phases becomes possible only if they minimize the quartic term $F^{(4)}$ of the free energy. Specifically, these transitions can occur once $T$ is lowered sufficiently to allow for the modulus of $M$ to grow, and the quartic part $F^{(4)}$ to overcome the SOC energy. At the temperature $T_{\mathrm{mag}}^{(2)}$, where this secondary transition takes place, the magnetic order reorganizes and develops additional components in order to minimize $F^{(4)}$ [93].

We proceed by providing the implications of a nonnegligible SOC on the phase diagrams depicted in Figs. 5(a), 5(c), and 6(a). The IC-MS region is unaffected by the addition of SOC. On the other hand, the IC CSDW and IC SVC are affected if $\alpha_{1,2}$ and $\alpha_{3}$ are the smallest, respectively. Starting with $\mathrm{BaFe}_{2} \mathrm{As}_{2}$ and $\alpha_{1}$ (or $\alpha_{2}$ ) being the smallest, we find that the free-energy hierarchy of Fig. 5(b) implies that the IC phases of Fig. 5(a), DPMH, IC CSDW, and $\mathrm{SWC}_{4}$, are all replaced by the IC-SVC phase, while the MH is replaced by the IC-MS phase. On the contrary, if $\alpha_{3}$ is the smallest, the IC-CSDW phase replaces the other IC phases, while the commensurate SVC phase is replaced by the commensurate CSDW phase. The phase diagram in the case of LaFeAsO, Fig. 5(c), is unchanged if $\alpha_{1}$ (or $\alpha_{2}$ ) is the smallest. On the other hand, if $\alpha_{3}$ is the smallest, the IC-MS phase is stabilized in the IC regions of the phase diagram, as can be gleaned from Fig. 5(d). In Fig. 6 , the $\mathrm{SWC}_{4}$ phase is replaced by the IC-SVC phase in the case where $\alpha_{1}$ (or $\left.\alpha_{2}\right)$ is the smallest. In the case where $\alpha_{3}$ is the smallest, the IC-CSDW phase replaces both the $\mathrm{SWC}_{4}$ and the IC-SVC phases. For $\langle n\rangle=5.77$, the IC-CSDW phase is nearly degenerate with the MS $\| \mathrm{MH}$ phase, and is thus hidden in Fig. 6.

Note that, while the phases to be replaced do not constitute leading instabilities in the presence of SOC, they still minimize the $F^{(4)}$ part of the free energy. As a result, additional magnetic-order-parameter components will appear at lower temperatures, possibly resulting in an admixture of phases [94]. In this manner, all the phases 
predicted by the spin-isotropic free energy are recovered through secondary phase transitions, provided we remain within the regime of weak SOC. We remark that the above analysis holds when one of the $\alpha_{1,2,3}$ coefficients is smaller than the other two. Otherwise, possible degeneracies between pairs of these coefficients can allow for textured phases to survive as leading instabilities or significantly narrow down the difference $\left|T_{\text {mag }}^{\mathrm{SOC}}-T_{\text {mag }}^{(2)}\right|$. Interestingly, the smaller this difference becomes, the more difficult it may be to experimentally distinguish the two transitions.

\section{Connection to the unknown $C_{2}$-symmetric magnetic phase of $\mathrm{Ba}_{1-x} \mathrm{Na}_{x} \mathrm{Fe}_{2} \mathrm{As}_{2}$}

Here we comment on the connection between our findings and the experimental results presented in Ref. [24]. In Ref. [24], an additional $C_{2}$-symmetric phase was observed, which was shown to be distinct from the usual MS phase. It is challenging to account for such a phase within the usual commensurate scenario. Indeed, there are no commensurate candidates resulting from second-order phase transitions from the paramagnetic phase. On the other hand, allowing for IC magnetism introduces six additional phases, five of which are $C_{2}$ symmetric. As discussed above, however, the presence of SOC also precludes the appearance of these phases as a result of a second-order phase transition from the paramagnetic phase. The experimental observations can be reconciled with the above facts provided that the magnetic transition is first order. In this case, the intermediate IC MS, IC SVC. or IC CSDW dictated by the finite SOC is no longer required, and a direct transition between the paramagnetic phase and one of the five new textured IC $\mathrm{C}_{2}$-symmetric phases is possible. At this stage, very little is known about the properties of the newly observed $C_{2}$-symmetric magnetic phase. Therefore, to resolve the nature of this phase additional experimental results are warranted, in particular to confirm whether the magnetic order is incommensurate in this region or not.

\section{CONCLUSIONS AND OUTLOOK}

In the present work we shed light on aspects of IC magnetism and provide a general classification of the possible magnetic phases that appear at the paramagnetic-magnetic transition for particular types of incommensurability. Our study is motivated by the marked tendency of the FeSCs towards magnetism and the observation of a puzzling $C_{2}$-symmetric phase, distinct from the magnetic stripe, in Na-doped $\mathrm{BaFe}_{2} \mathrm{As}_{2}$ [24].

By employing two realistic five-orbital models, we demonstrate that such IC scenarios are feasible in these systems. Our findings reveal that a subset of these new phases, consisting of the magnetic helix, double parallel magnetic helix, and the $C_{4}$-symmetric spin-whirl crystal, can emerge in the $\mathrm{BaFe}_{2} \mathrm{As}_{2}$ band structure upon electron or hole doping. On the other hand, an IC spin-vortex crystal phase can emerge on the substantially hole-doped side of the $\mathrm{LaFeAsO}$ phase diagram. A $C_{4}$-symmetric spin-whirl crystal phase is made possible in this case by employing orbital-dependent interactions.

To detect the IC phases studied here in FeSCs and other materials, suitable experimental methods have to be employed. Similar to the predictions of Ref. [95] for the three standard commensurate magnetic phases, experimental fingerprints of the novel IC phases are expected to become evident in spin-resolved scanning tunneling microscopy. However, since directly probing the spin structure of the order parameter by, e.g., polarized neutron scattering can be challenging, we propose alternative routes for diagnosing the underlying magnetic order. These indirect measurements rely on inferring the induced charge order and magnetoelectric coupling. The induced charge order is expected to be detectable via the observation of Bragg peaks at particular wave vectors from the set $\left\{ \pm 2 \boldsymbol{Q}_{1,2}, \pm \boldsymbol{Q}_{1} \pm \boldsymbol{Q}_{2}\right\}$, depending on the magnetic phase. The magnetic helix, the IC spin-vortex crystal, and the double parallel magnetic helix phases can be directly distinguished via this method. In contrast, the remaining six order parameters can be uniquely identified by complementary magnetoelectric measurements. Textured magnetic phases induce inversion-symmetry-breaking SOC that makes direct and inverse spin-galvanic effects accessible. In more detail, one can induce a ferromagnetic moment via a current flow or the application of an electric field, while reciprocal phenomena are also possible. The orientation of the induced magnetization is given by the spin vectors' cross products $i \hat{\boldsymbol{n}}_{s} \times \hat{\boldsymbol{n}}_{s}^{*}(s=1,2)$, while its appearance strictly depends on the orientation of the abovementioned external perturbations.

In addition to these features, the spin-whirl crystal phases are of particular interest, since they can acquire a nonzero Skyrmion charge via applying a Zeeman field, but more importantly, they can be employed for realizing intrinsic two-dimensional topological superconductors. The possible microscopic coexistence of textured phases with spinsinglet superconductivity opens the door for actualizing intrinsic topological systems harboring Majorana fermions [44,45,53-57]. The latter become accessible by virtue of the inversion-symmetry-breaking SOC and magnetoelectric effects induced by the textured phases. Hence, a transition from a topologically trivial to a nontrivial SC phase is expected to take place as the system enters the coexistence phase between IC magnetism and superconductivity. As we demonstrate, textured phases can be stabilized in FeSCs, with the most prominent for engineering topological superconductivity being the $C_{4}$-symmetric spin-whirl crystal phase, found as a stable minimum of the free energy for both band structure types considered here.

Interestingly, the IC nature of these magnetic textures implies that a nonzero ferromagnetic moment will be 
generated in a finite-sized two-dimensional FeSC. In the event that this net-magnetization field is felt by the entire bulk (only by the surface) of the FeSC material, the resulting magnetic superconductor will belong to the class of chiral (helical) topological superconductors. In the case of a gapped bulk energy spectrum, these magnetic superconductors are characterized by a $\mathbb{Z}\left(\mathbb{Z}_{2}\right)$ topological invariant $[39,44]$ that, as long as bulk-boundary correspondence remains intact [96], yields the number of chiral (helical) Majorana modes per edge. Instead, if the bulk energy spectrum exhibits nodal gap closings, then flatband or other more complex types of Majorana edge modes become accessible [82].

The potential experimental observation of microscopic coexistence of magnetism and superconductivity in FeSCs opens novel paths for crafting topological superconductors, distinct from the already existing mechanisms involving FeSe [64,86,97-99] or other hybrid structures consisting of two-dimensional magnetic textures [45,54-57] in proximity to conventional superconductors.

\section{ACKNOWLEDGMENTS}

The authors gratefully acknowledge D. Steffensen, D.D. Scherer, and M.N. Gastiasoro for inspiring and helpful discussions. M. H. C. and B. M. A. acknowledge financial support from a Lundbeckfond fellowship (Grant No. A9318). P. K. and B. M. A. acknowledge support from the Independent Research Fund Denmark Grant No. DFF-6108-00096.

\section{APPENDIX A: INCOMMENSURATE MAGNETIC PHASES}

As we show in the main text, there exist nine distinct incommensurate magnetic phases which extremize the Landau functional considered in Eq. (1). For each one of these phases we present the configuration of the respective $\hat{\boldsymbol{n}}_{1,2}$ spin vectors, the corresponding (or other symmetry-equivalent) magnetization profile $\boldsymbol{M}(\boldsymbol{r})$, and also the corresponding normalized and shifted quartic freeenergy term $\mathcal{F}^{(4)} \equiv 4 F^{(4)} / M^{4}-2 \tilde{\beta}$ (the quadratic term is the same for all phases).

\section{Incommensurate magnetic stripe}

This single- $Q C_{2}$-symmetric phase has a commensurate analog, appears only for $\beta-\tilde{\beta}<0$, and has the following characteristics:

$$
\begin{aligned}
\hat{\boldsymbol{n}}_{1} & =(0,0,1) \quad \text { and } \quad \hat{\boldsymbol{n}}_{2}=(0,0,0), \\
\boldsymbol{M}(\boldsymbol{r}) & =2 M\left(0,0, \cos \left(\boldsymbol{Q}_{1} \cdot \boldsymbol{r}\right)\right), \\
\mathcal{F}^{(4)} & =2(\beta-\tilde{\beta})
\end{aligned}
$$

\section{Incommensurate charge-spin density wave}

This double- $Q C_{4}$-symmetric phase has a commensurate analog, appears only for $\tilde{g}<0$, and is described by

$$
\begin{aligned}
\hat{\boldsymbol{n}}_{1} & =(0,0,1) \quad \text { and } \quad \hat{\boldsymbol{n}}_{2}=(0,0,1), \\
\boldsymbol{M}(\boldsymbol{r}) & =2 M\left(0,0, \cos \left(\boldsymbol{Q}_{1} \cdot \boldsymbol{r}\right)+\cos \left(\boldsymbol{Q}_{2} \cdot \boldsymbol{r}\right)\right), \\
\mathcal{F}^{(4)} & =2(\beta-\tilde{\beta}) \frac{G+\tilde{G}+1}{2} .
\end{aligned}
$$

\section{Incommensurate spin-vortex crystal}

This double- $Q C_{4}$-symmetric phase has a commensurate analog, appears only for $\tilde{g}>0$, and has the following form:

$$
\begin{aligned}
\hat{\boldsymbol{n}}_{1} & =(0,0,1) \quad \text { and } \quad \hat{\boldsymbol{n}}_{2}=(0,1,0), \\
\boldsymbol{M}(\boldsymbol{r}) & =2 M\left(0, \cos \left(\boldsymbol{Q}_{2} \cdot \boldsymbol{r}\right), \cos \left(\boldsymbol{Q}_{1} \cdot \boldsymbol{r}\right)\right), \\
\mathcal{F}^{(4)} & =2(\beta-\tilde{\beta}) \frac{G+1}{2} .
\end{aligned}
$$

\section{Magnetic helix}

This single- $Q C_{2}$-symmetric phase is new and appears only for $\beta-\tilde{\beta}>0$. One finds

$$
\begin{aligned}
\hat{\boldsymbol{n}}_{1} & =\frac{1}{\sqrt{2}}(i, 0,1) \quad \text { and } \quad \hat{\boldsymbol{n}}_{2}=(0,0,0), \\
\boldsymbol{M}(\boldsymbol{r}) & =2 M\left(\sin \left(\boldsymbol{Q}_{1} \cdot \boldsymbol{r}\right), 0, \cos \left(\boldsymbol{Q}_{1} \cdot \boldsymbol{r}\right)\right), \\
\mathcal{F}^{(4)} & =0 .
\end{aligned}
$$

\section{Incommensurate magnetic stripe and $\|$ magnetic helix}

This double- $\boldsymbol{Q} C_{2}$-symmetric phase is new and we obtain

$$
\begin{aligned}
\hat{\boldsymbol{n}}_{1}= & (0,0,1) \quad \text { and } \quad \hat{\boldsymbol{n}}_{2}=(i \sin \lambda, 0, \cos \lambda), \\
\mathcal{F}^{(4)}= & 2(\beta-\tilde{\boldsymbol{\beta}}) \frac{(\tilde{G}+2 G)^{2}}{(\tilde{G}+2)^{2}+8(G-1)}, \\
\boldsymbol{M}(\boldsymbol{r})= & 2 M\left(\sin \eta \sin \lambda \sin \left(\boldsymbol{Q}_{2} \cdot \boldsymbol{r}\right), 0, \cos \eta \cos \left(\boldsymbol{Q}_{1} \cdot \boldsymbol{r}\right)\right. \\
& \left.+\sin \eta \cos \lambda \cos \left(\boldsymbol{Q}_{2} \cdot \boldsymbol{r}\right)\right),
\end{aligned}
$$

with

$$
\cos (2 \eta)=-\frac{\tilde{G}^{2}-4}{(\tilde{G}+2)^{2}+8(G-1)}
$$

and

$$
\cos (2 \lambda)=-\tilde{G} \frac{2 G+\tilde{G}}{\tilde{G}(\tilde{G}+2)+4(G-1)} .
$$

Note that the helix becomes isotropic, i.e., $\lambda=\pi / 4$, only for $\tilde{G}=0$. 


\section{Incommensurate magnetic stripe and $\perp$ magnetic helix}

This double- $Q C_{2}$-symmetric phase is new and has the following features:

$$
\begin{aligned}
\hat{\boldsymbol{n}}_{1}= & (0,0,1) \quad \text { and } \quad \hat{\boldsymbol{n}}_{2}=\frac{1}{\sqrt{2}}(i, 1,0), \\
\boldsymbol{M}(\boldsymbol{r})= & \sqrt{2} M\left(\sin \eta \sin \left(\boldsymbol{Q}_{2} \cdot \boldsymbol{r}\right), \sin \eta \cos \left(\boldsymbol{Q}_{2} \cdot \boldsymbol{r}\right),\right. \\
& \left.\sqrt{2} \cos \eta \cos \left(\boldsymbol{Q}_{1} \cdot \boldsymbol{r}\right)\right), \\
\mathcal{F}^{(4)}= & 2(\beta-\tilde{\beta}) \frac{G^{2}}{2 G-1}, \quad \text { with } \quad \cos (2 \eta)=\frac{1}{2 G-1} .
\end{aligned}
$$

\section{Douple parallel magnetic helix}

This double- $Q C_{2}$-symmetric phase (in spite of $\eta=\pi / 4$ ) is new, appears for $\tilde{G} \neq \pm 2$, and is described by

$$
\begin{aligned}
\hat{\boldsymbol{n}}_{1,2}= & \frac{1}{\sqrt{2}}(i, 0,1), \\
\boldsymbol{M}(\boldsymbol{r})= & M\left(\sin \left(\boldsymbol{Q}_{1} \cdot \boldsymbol{r}\right)+\sin \left(\boldsymbol{Q}_{2} \cdot \boldsymbol{r}\right), 0, \cos \left(\boldsymbol{Q}_{1} \cdot \boldsymbol{r}\right)\right. \\
& \left.+\cos \left(\boldsymbol{Q}_{2} \cdot \boldsymbol{r}\right)\right), \\
\mathcal{F}^{(4)}= & 2(\beta-\tilde{\beta}) \frac{G+\tilde{G} / 2}{2} .
\end{aligned}
$$

\section{8. $C_{4}$-symmetric spin-whirl crystal}

This double- $Q C_{4}$-symmetric phase is new, appears for $\tilde{G}(1-G)+4 G \neq 0$, and one finds:

$$
\begin{aligned}
\hat{\boldsymbol{n}}_{1}= & (i \cos \lambda, 0, \sin \lambda) \quad \text { and } \hat{\boldsymbol{n}}_{2}=(0, i \cos \lambda, \sin \lambda), \\
\mathcal{F}^{(4)}= & 2(\beta-\tilde{\boldsymbol{\beta}}) \frac{G(\tilde{G}+4)+\tilde{G}}{2(\tilde{G}+4)}, \\
\boldsymbol{M}(\boldsymbol{r})= & \sqrt{2} M\left(\cos \lambda \sin \left(\boldsymbol{Q}_{1} \cdot \boldsymbol{r}\right), \cos \lambda \sin \left(\boldsymbol{Q}_{2} \cdot \boldsymbol{r}\right),\right. \\
& \left.\sin \lambda \cos \left(\boldsymbol{Q}_{1} \cdot \boldsymbol{r}\right)+\sin \lambda \cos \left(\boldsymbol{Q}_{2} \cdot \boldsymbol{r}\right)\right),
\end{aligned}
$$

with $\cos (2 \lambda)=\tilde{G} /(\tilde{G}+4)$. The latter implies $\cos (2 \eta)=$ $0 \Rightarrow \eta=\pi / 4$. Note also that for $\tilde{g}, \tilde{G}=0$, we obtain $\cos (2 \lambda)=0 \Rightarrow \lambda=\pi / 4$, and leads to a symmetric double- $\boldsymbol{Q}$ noncoplanar $C_{4}$-phase $\boldsymbol{M}(\boldsymbol{r})=M\left(\sin \left(\boldsymbol{Q}_{1} \cdot \boldsymbol{r}\right)\right.$, $\left.\sin \left(\boldsymbol{Q}_{2} \cdot \boldsymbol{r}\right), \cos \left(\boldsymbol{Q}_{1} \cdot \boldsymbol{r}\right)+\cos \left(\boldsymbol{Q}_{2} \cdot \boldsymbol{r}\right)\right)$.

\section{9. $C_{2}$-symmetric spin-whirl crystal}

This new double- $Q C_{2}$-symmetric phase consists of an isotropic magnetic helix for $\boldsymbol{Q}_{1}$ coexisting with an anisotropic magnetic helix for $\boldsymbol{Q}_{2}$ :

$$
\begin{aligned}
\hat{\boldsymbol{n}}_{1} & =\frac{1}{\sqrt{2}}(i, 0,1) \quad \text { and } \quad \hat{\boldsymbol{n}}_{2}=(0, i \sin \lambda, \cos \lambda), \\
\mathcal{F}^{(4)} & =2(\beta-\tilde{\beta}) \frac{(\tilde{G}+4 G)^{2}}{(\tilde{G}+4)^{2}+16(2 G-1)}, \\
\boldsymbol{M}(\boldsymbol{r}) & =2 M\left(\frac{\cos \eta}{\sqrt{2}} \sin \left(\boldsymbol{Q}_{1} \cdot \boldsymbol{r}\right), \sin \eta \sin \lambda \sin \left(\boldsymbol{Q}_{2} \cdot \boldsymbol{r}\right),\right. \\
& \left.\frac{\cos \eta}{\sqrt{2}} \cos \left(\boldsymbol{Q}_{1} \cdot \boldsymbol{r}\right)+\sin \eta \cos \lambda \cos \left(\boldsymbol{Q}_{2} \cdot \boldsymbol{r}\right)\right),
\end{aligned}
$$

with

$$
\cos (2 \eta)=-\frac{\tilde{G}^{2}}{(\tilde{G}+4)^{2}+16(2 G-1)}
$$

and

$$
\cos (2 \lambda)=-\tilde{G} \frac{\tilde{G}+4 G}{\tilde{G}(\tilde{G}+4)+16 G}
$$

\section{APPENDIX B: INDUCED CHARGE ORDER}

Here we derive the coupling term between magnetic and charge order. For simplicity, and without loss of generality, let us restrict ourselves to be near the magnetic critical temperature, for which an expansion in terms of the magnetic order parameters and the charge density is permissible. We will further assume that the orbital weight $\hat{v}_{\boldsymbol{q}}$ of the magnetic order parameters $\hat{\boldsymbol{M}}_{\boldsymbol{q}}$ is fixed by the spin susceptibility, i.e., $\hat{\boldsymbol{M}}_{\boldsymbol{q}}=\boldsymbol{M}_{q} \hat{v}_{\boldsymbol{q}}$ (see Sec. VI A).

We start from a general multiorbital model Hamiltonian for an itinerant system, rewritten in $\boldsymbol{k}$ space and within the framework of second quantization: $\mathcal{H}_{0}=\sum_{k} \boldsymbol{\psi}_{\boldsymbol{k}}^{\dagger} \hat{\mathbf{1}}_{2} \otimes$ $\hat{\mathcal{H}}_{0}(\boldsymbol{k}) \boldsymbol{\psi}_{\boldsymbol{k}}$, where we introduced the electron creation and annihilation operators acting in orbital and spin spaces. Note that $\hat{\mathcal{H}}_{0}(\boldsymbol{k})$ is a matrix in orbital space, and the complete Hamiltonian is diagonal in spin space, since SOC has been omitted from the outset. We then add the term

$$
\mathcal{V}=\sum_{k, q} \boldsymbol{\psi}_{k+\boldsymbol{q}}^{\dagger}\left(\boldsymbol{M}_{\boldsymbol{q}} \cdot \boldsymbol{\sigma} \otimes \hat{v}_{\boldsymbol{q}}+\rho_{\boldsymbol{q}} \hat{\mathbf{1}}_{2} \otimes \hat{\mathbf{1}}_{5}\right) \boldsymbol{\psi}_{\boldsymbol{k}}
$$

and perform a perturbative expansion of the resulting free energy to the lowest allowed coupling between charge and magnetic order terms. The lowest-order coupling term appears at cubic order of perturbation and the corresponding free-energy contribution is

$$
\begin{aligned}
F= & \frac{1}{3 \beta} \sum_{\boldsymbol{q}, \boldsymbol{p}} \sum_{i k_{n}, \boldsymbol{k}} \operatorname{Tr}\left[\hat{\mathbf{1}}_{2} \otimes \hat{\mathcal{G}}_{0}\left(i k_{n}, \boldsymbol{k}\right) \hat{\mathcal{V}}_{\boldsymbol{q}}\right. \\
& \left.\times \hat{\mathbf{1}}_{2} \otimes \hat{\mathcal{G}}_{0}\left(i k_{n}, \boldsymbol{k}-\boldsymbol{q}\right) \hat{\mathcal{V}}_{\boldsymbol{p}} \hat{\mathbf{1}}_{2} \otimes \hat{\mathcal{G}}_{0}\left(i k_{n}, \boldsymbol{k}-\boldsymbol{q}-\boldsymbol{p}\right) \hat{\mathcal{V}}_{-\boldsymbol{q}-\boldsymbol{p}}\right]
\end{aligned}
$$


with the trace being over spin and orbital indices, $1 / \beta=k_{B} T, k_{n}$ denotes the fermionic Matsubara frequencies, $\hat{\mathcal{G}}_{0}\left(i k_{n}, \boldsymbol{k}\right)=\left[i k_{n} \hat{\mathbf{1}}_{5}-\hat{\mathcal{H}}_{0}(\boldsymbol{k})\right]^{-1}$ defines the bare Matsubara orbital space matrix Green's function, and $\hat{\mathcal{V}}_{\boldsymbol{q}} \equiv \boldsymbol{M}_{\boldsymbol{q}} \cdot \boldsymbol{\sigma} \otimes \hat{v}_{\boldsymbol{q}}+\rho_{\boldsymbol{q}} \hat{\mathbf{I}}_{2} \otimes \hat{\mathbf{I}}_{5}$. By carrying out the trace over the spin degrees of freedom and after taking into account all the products of the interaction terms, we have the general result:

$$
\begin{aligned}
F= & \sum_{\boldsymbol{q}, \boldsymbol{p}= \pm \boldsymbol{Q}_{1,2}}\left\{\frac { 2 } { \beta } \sum _ { i k _ { n } , \boldsymbol { k } } \operatorname { T r } _ { \text { orbital } } \left[\hat{\mathcal{G}}_{0}\left(i k_{n}, \boldsymbol{k}\right) \hat{v}_{\boldsymbol{q}} \hat{\mathcal{G}}_{0}\left(i k_{n}, \boldsymbol{k}-\boldsymbol{q}\right)\right.\right. \\
& \left.\left.\times \hat{v}_{\boldsymbol{p}} \hat{\mathcal{G}}_{0}\left(i k_{n}, \boldsymbol{k}-\boldsymbol{q}-\boldsymbol{p}\right)\right]\right\}\left|\boldsymbol{M}_{\boldsymbol{q}}\right|\left|\boldsymbol{M}_{\boldsymbol{p}}\right| \hat{\boldsymbol{n}}_{\boldsymbol{q}} \cdot \hat{\boldsymbol{n}}_{\boldsymbol{p}} \rho_{-(\boldsymbol{q}+\boldsymbol{p})} .
\end{aligned}
$$

Therefore, we can obtain induced charge order for the set of wave vectors $\left\{ \pm 2 \boldsymbol{Q}_{1,2}, \pm\left(\boldsymbol{Q}_{1} \pm \boldsymbol{Q}_{2}\right)\right\}$, depending on which spin-vector inner products become nonzero. Note that there exist additional higher-order contributions to the free energy that yield (i) corrections to the above and (ii) charge order at additional wave vectors. However, these contributions will lead to Bragg peaks with lower intensity compared to the one found at cubic order, and thus can be experimentally differentiated.

\section{APPENDIX C: MAGNETOELECTRIC EFFECTS}

The aim of this section is to illustrate the emergence of the magnetoelectric effects discussed in the main text. The $\boldsymbol{q}=\mathbf{0}$ ferromagnetic component of the magnetization $\boldsymbol{M}_{\mathbf{0}}$ couples to a generally time-dependent electromagnetic vector potential $\boldsymbol{A}$, and generates an electric field $(\mathcal{E})$ or current $(\boldsymbol{I})$. The coupling of the itinerant system to the vector potential will be restricted to the paramagnetic contribution, sufficient for the lowest-order coupling with respect to $\boldsymbol{A}$ that is pursued here. Therefore, we employ a similar approach as in the section above and focus near the magnetic critical temperature, where the magnetic order parameters can be treated perturbatively. Then we add to the Hamiltonian, in the Schrödinger picture, the perturbation term

$$
\begin{aligned}
\mathcal{V}= & \sum_{\boldsymbol{k}, \boldsymbol{q}} \boldsymbol{\psi}_{\boldsymbol{k}+\boldsymbol{q}}^{\dagger}\left\{\boldsymbol{M}_{\boldsymbol{q}} \cdot \boldsymbol{\sigma} \otimes \hat{v}_{\boldsymbol{q}}+\left[\boldsymbol{M}_{\mathbf{0}}(t) \cdot \boldsymbol{\sigma} \otimes \hat{\mathbf{1}}_{5}+\frac{e}{\hbar} \hat{\mathbf{1}}_{2}\right.\right. \\
& \left.\left.\otimes \frac{\partial \hat{\mathcal{H}}_{0}(\boldsymbol{k})}{\partial \boldsymbol{k}} \cdot \boldsymbol{A}(t)\right] \delta_{\boldsymbol{q}, \boldsymbol{0}}\right\} \boldsymbol{\psi}_{\boldsymbol{k}}
\end{aligned}
$$

with $e>0$. We thus perform a perturbative expansion of the resulting free energy to the lowest allowed coupling between the vector potential, the ferromagnetic moment, and the $\boldsymbol{q}= \pm \boldsymbol{Q}_{1,2}$ magnetic order terms. The lowest-order coupling term appears at quartic order of the perturbation term, and the corresponding free-energy contribution reads

$$
\begin{aligned}
F= & \frac{1}{4 \beta} \sum_{k, q_{1,2,3}} \operatorname{Tr}\left[\hat{\mathbf{1}}_{2} \otimes \hat{\mathcal{G}}_{0}(k) \hat{\mathcal{V}}_{q_{1}} \hat{\mathbf{1}}_{2} \otimes \hat{\mathcal{G}}_{0}\left(k-q_{1}\right) \hat{\mathcal{V}}_{q_{2}}\right. \\
& \times \hat{\mathbf{1}}_{2} \otimes \hat{\mathcal{G}}_{0}\left(k-q_{1}-q_{2}\right) \hat{\mathcal{V}}_{q_{3}} \hat{\mathbf{1}}_{2} \otimes \hat{\mathcal{G}}_{0}\left(k-q_{1}-q_{2}-q_{3}\right) \\
& \left.\times \hat{\mathcal{V}}_{-q_{1}-q_{2}-q_{3}}\right],
\end{aligned}
$$

where we introduced the four vectors $k=\left(i k_{n}, \boldsymbol{k}\right)$ and $q=\left(i \omega_{s}, \boldsymbol{q}\right)$, where the latter consists of the bosonic Matsubara frequency $i \omega_{s}$ and wave vector $\boldsymbol{q}$. The respective perturbation terms read

$$
\begin{aligned}
\hat{\mathcal{V}}_{q} \equiv & \boldsymbol{M}_{\boldsymbol{q}} \cdot \boldsymbol{\sigma} \otimes \hat{v}_{\boldsymbol{q}} \delta_{\omega_{s}, 0}+\left[\boldsymbol{M}_{\mathbf{0}}\left(i \omega_{s}\right) \cdot \boldsymbol{\sigma} \otimes \hat{\mathbf{1}}_{5}\right. \\
& \left.+\frac{e}{\hbar} \hat{\mathbf{1}}_{2} \otimes \frac{\partial \hat{\mathcal{H}}_{0}(\boldsymbol{k})}{\partial \boldsymbol{k}} \cdot \boldsymbol{A}\left(i \omega_{s}\right)\right] \delta_{\boldsymbol{q}, \boldsymbol{0}} .
\end{aligned}
$$

For the itinerant systems of interest, inversion symmetry is present; therefore, $\hat{\mathcal{H}}_{0}(\boldsymbol{k})=\hat{\mathcal{H}}_{0}(-\boldsymbol{k})$ and $\partial \hat{\mathcal{H}}_{0}(\boldsymbol{k}) / \partial k_{a}=$ $-\partial \hat{\mathcal{H}}_{0}(-\boldsymbol{k}) / \partial k_{a}$. As a result, we find that the magnetoelectric coupling can be nonzero only when $\boldsymbol{A} \| \hat{\boldsymbol{q}}$, with $\hat{\boldsymbol{q}}=\boldsymbol{q} /|\boldsymbol{q}|$. By tracing over the spin indices and after taking into account all the possible combinations, we obtain:

$$
\begin{aligned}
F= & \frac{e}{\hbar} \sum_{\omega_{s}} \sum_{\boldsymbol{q}= \pm \boldsymbol{Q}_{1,2}}\left|\boldsymbol{M}_{\boldsymbol{q}}\right|^{2} \boldsymbol{M}_{\mathbf{0}}\left(-i \omega_{s}\right) \cdot\left(i \hat{\boldsymbol{n}}_{\boldsymbol{q}} \times \hat{\boldsymbol{n}}_{\boldsymbol{q}}^{*}\right) \hat{\boldsymbol{q}} \cdot \boldsymbol{A}\left(i \omega_{s}\right) \frac{2}{\beta} \sum_{k_{n}, \boldsymbol{k}} \\
& \times\left\{\operatorname{Tr}_{\text {orbital }}\left[\hat{\mathcal{G}}_{0}\left(i k_{n}, \boldsymbol{k}\right) \hat{\boldsymbol{q}} \cdot \frac{\partial \hat{\mathcal{H}}_{0}(\boldsymbol{k})}{\partial \boldsymbol{k}} \hat{\mathcal{G}}_{0}\left(i k_{n}-i \omega_{s}, \boldsymbol{k}\right) \hat{\mathcal{G}}_{0}\left(i k_{n}, \boldsymbol{k}\right) \hat{v}_{\boldsymbol{q}} \hat{\mathcal{G}}_{0}\left(i k_{n}, \boldsymbol{k}-\boldsymbol{q}\right) \hat{v}_{-\boldsymbol{q}}\right]\right. \\
& -\operatorname{Tr}_{\text {orbital }}\left[\hat{\mathcal{G}}_{0}\left(i k_{n}, \boldsymbol{k}\right) \hat{\boldsymbol{q}} \cdot \frac{\partial \hat{\mathcal{H}}_{0}(\boldsymbol{k})}{\partial \boldsymbol{k}} \hat{\mathcal{G}}_{0}\left(i k_{n}-i \omega_{s}, \boldsymbol{k}\right) \hat{v}_{\boldsymbol{q}} \hat{\mathcal{G}}_{0}\left(i k_{n}-i \omega_{s}, \boldsymbol{k}-\boldsymbol{q}\right) \hat{\mathcal{G}}_{0}\left(i k_{n}, \boldsymbol{k}-\boldsymbol{q}\right) \hat{v}_{-\boldsymbol{q}}\right] \\
& \left.+\operatorname{Tr}_{\text {orbital }}\left[\hat{\mathcal{G}}_{0}\left(i k_{n}, \boldsymbol{k}\right) \hat{\boldsymbol{q}} \cdot \frac{\partial \hat{\mathcal{H}}_{0}(\boldsymbol{k})}{\partial \boldsymbol{k}} \hat{\mathcal{G}}_{0}\left(i k_{n}-i \omega_{s}, \boldsymbol{k}\right) \hat{v}_{\boldsymbol{q}} \hat{\mathcal{G}}_{0}\left(i k_{n}-i \omega_{s}, \boldsymbol{k}-\boldsymbol{q}\right) \hat{v}_{-q} \hat{\mathcal{G}}_{0}\left(i k_{n}-i \omega_{s}, \boldsymbol{k}\right)\right]\right\} .
\end{aligned}
$$


In order to investigate the arising magnetoelectric response, one has to perform the analytical continuation, $i \omega_{s} \rightarrow$ $\omega+i 0^{+}$, to the real frequencies $\omega$.

\section{APPENDIX D: HUBBARD-HUND MODEL, MAGNETIC ORDER PARAMETERS, AND ORBITAL STRUCTURE FOR THE CASE OF IRON-BASED SUPERCONDUCTORS}

The magnetic transition temperature is obtained by identifying the first zero eigenvalue of the static part of the inverse magnetic propagator matrix when evaluated as a function of temperature and $\boldsymbol{q}$. The latter is defined as $\check{\mathcal{D}}_{\text {mag }}^{-1}(\boldsymbol{q})=\check{U}^{-1}-\check{\chi}_{0}(\boldsymbol{q})$, with "denoting rank-4 tensors in orbital space. Note that for our calculations we neglect the Hartree shift of the chemical potential induced by interactions. The orbital weight of the magnetic order parameter is obtained from the eigenmatrix associated with the zero eigenvalue, i.e., $\check{\mathcal{D}}_{\text {mag }}^{-1}\left(\boldsymbol{Q}_{1,2}\right) \hat{v}_{1,2}=\hat{0}$. As incommensurability sets in, the orbital content acquires imaginary parts; e.g., for $\mathrm{LaFeAsO}$ we find [in the basis $\left(x z, y z, x y, x^{2}-y^{2}, z^{2}\right)$ ]

$$
\hat{v}_{1}=\left(\begin{array}{ccccc}
0.395 & 0 & i 0.045 & 0 & 0 \\
0 & 0.501 & 0 & i 0.028 & -i 0.025 \\
i 0.045 & 0 & 0.574 & 0 & 0 \\
0 & i 0.028 & 0 & 0.335 & -0.038 \\
0 & -i 0.025 & 0 & -0.038 & 0.377
\end{array}\right) \text { and } \hat{v}_{2}=\left(\begin{array}{ccccc}
0.501 & 0 & 0 & i 0.028 & i 0.025 \\
0 & 0.395 & -i 0.045 & 0 & 0 \\
0 & -i 0.045 & 0.574 & 0 & 0 \\
i 0.028 & 0 & 0 & 0.335 & 0.038 \\
i 0.025 & 0 & 0 & 0.038 & 0.377
\end{array}\right) \text {, }
$$

where $\hat{v}_{1}$ is plotted in Fig. 4(l) in the main text. The quartic coefficients of the free energy are computed by performing a Hubbard-Stratonovich decoupling in the magnetic channel and expanding the trace-log to fourth order in the magnetic order parameters. The expression is truncated by assuming that only the lowest harmonics contribute. This is justified by comparing the magnitude of the peak at $\boldsymbol{Q}_{1,2}$ in the bare susceptibility with the peaks at higher integer multiples of $\boldsymbol{Q}_{1,2}$, as shown in Figs. 4(d) and 4(j). The quartic coefficients are rank- 8 tensors in orbital space, and to determine the magnetic order at the instability the coefficients are projected onto the leading instability using the orbital content provided by the above eigenmatrices.

The expressions for the quartic coefficients of the free energy are related to the microscropic band structure through

$$
\begin{aligned}
\tilde{\beta}_{1}^{a b c d e f g h}= & \frac{1}{16 \beta \mathcal{N}} \sum_{k}\left[G^{a b} G_{1}^{c d} G^{e f} G_{1}^{g h}+G^{e b} G_{1}^{c h} G^{a f} G_{1}^{g d}+2 G^{g b} G_{1}^{c d} G^{e h} G_{-1}^{a f}-2 G^{g b} G_{1}^{c h} G^{a d} G_{-1}^{e f}\right], \\
\tilde{\beta}_{2}^{a b c d e f g h}= & \frac{1}{16 \beta \mathcal{N}} \sum_{k}\left[G^{a b} G_{2}^{c d} G^{e f} G_{2}^{g h}+G^{e b} G_{2}^{c h} G^{a f} G_{2}^{g d}+2 G^{g b} G_{2}^{c d} G^{e h} G_{-2}^{a f}-2 G^{g b} G_{2}^{c h} G^{a d} G_{-2}^{e f}\right], \\
\left(\beta_{1}-\tilde{\beta}_{1}\right)^{a b c d e f g h}= & \frac{1}{16 \beta \mathcal{N}} \sum_{k}\left[-G^{a b} G_{1}^{c f} G^{g d} G_{1}^{e h}+2 G^{e b} G_{1}^{c f} G^{g h} G_{-1}^{a d}\right], \\
\left(\beta_{2}-\tilde{\beta}_{2}\right)^{a b c d e f g h}= & \frac{1}{16 \beta \mathcal{N}} \sum_{k}\left[-G^{a b} G_{2}^{c f} G^{g d} G_{2}^{e h}+2 G^{e b} G_{2}^{c f} G^{g h} G_{-2}^{a d}\right], \\
g^{a b c d e f g h}= & \frac{1}{8 \beta \mathcal{N}} \sum_{k}\left[G^{a b} G_{1}^{c d} G^{e f} G_{2}^{g h}+G^{g b} G_{1}^{c d} G^{e h} G_{-2}^{a f}+G^{a d} G_{-1}^{e b} G^{c f} G_{2}^{g h}+G^{g d} G_{-1}^{e b} G^{c h} G_{-2}^{a f}\right. \\
& \left.-G^{e f} G_{2}^{g b} G_{1+2}^{c h} G_{1}^{a d}-G^{c h} G_{1}^{a d} G_{1+2}^{e f} G_{2}^{g b}\right], \\
& \frac{1}{8 \beta \mathcal{N}} \sum_{k}\left[-G^{a b} G_{1}^{c f} G^{g d} G_{2}^{e h}+G^{e b} G_{1}^{c f} G^{g h} G_{-2}^{a d}+G^{a f} G_{-1}^{g b} G^{c d} G_{2}^{e h}-G^{e f} G_{-1}^{g b} G^{c h} G_{-2}^{a d}\right. \\
& \left.+G^{g d} G_{2}^{e b} G_{1+2}^{c h} G_{1}^{a f}+G^{a b} G_{1}^{c d} G_{1+2}^{e f} G_{2}^{g h}\right],
\end{aligned}
$$




$$
\begin{aligned}
\tilde{g}_{2}^{a b c d e f g h}= & \frac{1}{8 \beta \mathcal{N}} \sum_{k}\left[G^{e b} G_{1}^{c f} G^{g h} G_{2}^{a d}-G^{a b} G_{1}^{c f} G^{g d} G_{-2}^{e h}-G^{e f} G_{-1}^{g b} G^{c h} G_{2}^{a d}+G^{a f} G_{-1}^{g b} G^{c d} G_{-2}^{e h}\right. \\
& \left.+G^{g h} G_{2}^{a b} G_{1+2}^{c d} G_{1}^{e f}+G^{e b} G_{1}^{c h} G_{1+2}^{a f} G_{2}^{g d}\right]
\end{aligned}
$$

with $\mathcal{N}$ denoting the number of sites in $\boldsymbol{k}$ space. Here, $k=\left(i k_{n}, \boldsymbol{k}\right)$ and $G_{1,2}^{a b}$ is the fermionic Green function centered at $\boldsymbol{Q}_{1,2}$ :

$$
G_{1,2}^{a b}=\sum_{\nu} \frac{u_{\nu}^{a}\left(\boldsymbol{k}+\boldsymbol{Q}_{1,2}\right) u_{\nu}^{b}\left(\boldsymbol{k}+\boldsymbol{Q}_{1,2}\right)^{*}}{i k_{n}-E_{\nu}\left(\boldsymbol{k}+\boldsymbol{Q}_{1,2}\right)},
$$

with $k_{n}$ a fermionic Matsubara frequency. In the above we also employed the shorthand notation $f_{1+2} \equiv f_{\boldsymbol{Q}_{1}+\boldsymbol{Q}_{2}}$.

Note that the tensorial coefficients, e.g., $\tilde{\beta}_{1}^{\text {abcdefgh }}$ and $\tilde{\beta}_{2}^{a b c d e f g h}$, are not identical but are related by $C_{4}$ rotations. To proceed we project these onto the leading magnetic instability utilizing the orbital content provided by the matrices $v_{a b}\left(\boldsymbol{Q}_{1,2}\right)$ as in Ref. [11]; for instance, we have

$$
\begin{aligned}
\beta_{1}-\tilde{\boldsymbol{\beta}}_{1} \equiv & \sum_{\substack{a b c d \\
\text { efgh }}}\left(\beta_{1}-\tilde{\boldsymbol{\beta}}_{1}\right)^{a b c d e f g h} v_{a b}\left(\boldsymbol{Q}_{1}\right) \\
& \times v_{c d}\left(\boldsymbol{Q}_{1}\right) v_{e f}^{*}\left(\boldsymbol{Q}_{1}\right) v_{g h}^{*}\left(\boldsymbol{Q}_{1}\right) .
\end{aligned}
$$

Note that the contracted coefficients, such as $\beta_{1}-\tilde{\beta}_{1}$ and $\beta_{2}-\tilde{\beta}_{2}$, are identical.

[1] J. M. Allred, K. M. Taddei, D. E. Bugaris, M. J. Krogstad, S. H. Lapidus, D. Y. Chung, H. Claus, M. G. Kanatzidis, D. E. Brown, J. Kang, R. M. Fernandes, I. Eremin, S. Rosenkranz, O. Chmaissem, and R. Osborn, Double- $Q$ Spin-Density Wave in Iron Arsenide Superconductors, Nat. Phys. 12, 493 (2016).

[2] W. R. Meier, Q.-P. Ding, A. Kreyssig, S. L. Bud'ko, A. Sapkota, K. Kothapalli, V. Borisov, R. Valent, C. D. Batista, P. P. Orth, R. M. Fernandes, A. I. Goldman, Y. Furukawa, A. E. Böhmer, and P. C. Canfield, Hedgehog Spin-Vortex Crystal Stabilized in a Hole-Doped Iron-Based Superconductor., npj Quantum Mater. 3, 5 (2018).

[3] J. Lorenzana, G. Seibold, C. Ortix, and M. Grilli, Competing Orders in FeAs Layers, Phys. Rev. Lett. 101, 186402 (2008).

[4] I. Eremin and A. V. Chubukov, Magnetic Degeneracy and Hidden Metallicity of the Spin-Density-Wave State in Ferropnictides, Phys. Rev. B 81, 024511 (2010).

[5] P. M. R. Brydon, J. Schmiedt, and C. Timm, Microscopically Derived Ginzburg-Landau Theory for Magnetic Order in the Iron Pnictides, Phys. Rev. B 84, 214510 (2011).

[6] G. Giovannetti, C. Ortix, M. Marsman, M. Capone, J. van den Brink, and J. Lorenzana, Proximity of Iron Pnictide Superconductors to a Quantum Tricritical Point, Nat. Commun. 2, 398 (2011).
[7] M. N. Gastiasoro and B. M. Andersen, Competing Magnetic Double-Q Phases and Superconductivity-Induced Reentrance of $\mathrm{C}_{2}$ Magnetic Stripe Order in Iron Pnictides, Phys. Rev. B 92, 140506(R) (2015).

[8] X. Wang, J. Kang, and R. M. Fernandes, Magnetic Order without Tetragonal-Symmetry-Breaking in Iron Arsenides: Microscopic Mechanism and Spin-Wave Spectrum, Phys. Rev. B 91, 024401 (2015).

[9] J. Kang, X. Wang, A. V. Chubukov, and R. M. Fernandes, Interplay between Tetragonal Magnetic Order, Stripe Magnetism, and Superconductivity in Iron-Based Materials, Phys. Rev. B 91, 121104(R) (2015).

[10] M. H. Christensen, J. Kang, B. M. Andersen, I. Eremin, and R. M. Fernandes, Spin Reorientation Driven by the Interplay between Spin-Orbit Coupling and Hund's Rule Coupling in Iron Pnictides, Phys. Rev. B 92, 214509 (2015).

[11] M. H. Christensen, D. D. Scherer, P. Kotetes, and B. M. Andersen, Role of Multiorbital Effects in the Magnetic Phase Diagram of Iron-Pnictides, Phys. Rev. B 96, 014523 (2017).

[12] D. D. Scherer, I. Eremin, and B. M. Andersen, Collective Magnetic Excitations of $\mathrm{C}_{4}$-Symmetric Magnetic States in Iron-Based Superconductors, Phys. Rev. B 94, 180405(R) (2016).

[13] S. Avci, O. Chmaissem, J. M. Allred, S. Rosenkranz, I. Eremin, A. V. Chubukov, D. E. Bugaris, D. Y. Chung, M. G. Kanatzidis, J.-P Castellan, J. A. Schlueter, H. Claus, D. D. Khalyavin, P. Manuel, A. Daoud-Aladine, and R. Osborn, Magnetically Driven Suppression of Nematic Order in an Iron-Based Superconductor, Nat. Commun. 5, 3845 (2014).

[14] F. Waßer, A. Schneidewind, Y. Sidis, S. Wurmehl, S. Aswartham, B. Büchner, and M. Braden, Spin Reorientation in $\mathrm{Ba}_{0.65} \mathrm{Na}_{0.35} \mathrm{Fe}_{2} \mathrm{As}_{2}$ Studied by Single-Crystal Neutron Diffraction, Phys. Rev. B 91, 060505 (2015).

[15] E. Hassinger, G. Gredat, F. Valade, S. R. de Cotret, A. Juneau-Fecteau, J.-P. Reid, H. Kim, M. A. Tanatar, R. Prozorov, B. Shen, H.-H. Wen, N. Doiron-Leyraud, and L. Taillefer, Pressure-Induced Fermi-Surface Reconstruction in the Iron-Arsenide Superconductor $\mathrm{Ba}_{1-x} \mathrm{~K}_{x} \mathrm{Fe}_{2} \mathrm{As}_{2}$ : Evidence of a Phase Transition inside the Antiferromagnetic Phase, Phys. Rev. B 86, 140502 (2012).

[16] A. E. Böhmer, F. Hardy, L. Wang, T. Wolf, P. Schweiss, and C. Meingast, Superconductivity-Induced Re-entrance of the Orthorhombic Distortion in $\mathrm{Ba}_{1 x} \mathrm{~K}_{x} \mathrm{Fe}_{2} \mathrm{As}_{2}$, Nat. Commun. 6, 7911 (2015).

[17] J. M. Allred, S. Avci, Y. Chung, H. Claus, D. D. Khalyavin, P. Manuel, K. M. Taddei, M. G. Kanatzidis, S. Rosenkranz, R. Osborn, and O. Chmaissem, Tetragonal Magnetic Phase in $\mathrm{Ba}_{1-x} \mathrm{~K}_{x} \mathrm{Fe}_{2} \mathrm{As}_{2}$ from $X$-Ray and Neutron Diffraction, Phys. Rev. B 92, 094515 (2015). 
[18] Y. Zheng, P. M. Tam, J. Hou, A. E. Böhmer, T. Wolf, C. Meingast, and R. Lortz, Absence of Nematic Order in the Pressure-Induced Intermediate Phase of the Iron-Based Superconductor $\mathrm{Ba}_{0.85} \mathrm{~K}_{0.15} \mathrm{Fe}_{2} \mathrm{As}_{2}$, Phys. Rev. B 93, 104516 (2016).

[19] B. P. P. Mallett, Y. G. Pashkevich, A. Gusev, T. Wolf, and C. Bernhard, Muon Spin Rotation Study of the Magnetic Structure in the Tetragonal Antiferromagnetic State of Weakly Underdoped $\mathrm{Ba}_{1-x} \mathrm{~K}_{x} \mathrm{Fe}_{2} \mathrm{As}_{2}$, Europhys. Lett. 111, 57001 (2015).

[20] B. P. P. Mallett, P. Marsik, M. Yazdi-Rizi, T. Wolf, A. E. Böhmer, F. Hardy, C. Meingast, D. Munzar, and C. Bernhard, Infrared Study of the Spin Reorientation Transition and Its Reversal in the Superconducting State in Underdoped $\mathrm{Ba}_{1-x} \mathrm{~K}_{x} \mathrm{Fe}_{2} \mathrm{As}_{2}$, Phys. Rev. Lett. 115, 027003 (2015).

[21] D. K. Pratt, M. G. Kim, A. Kreyssig, Y. B. Lee, G. S. Tucker, A. Thaler, W. Tian, J. L. Zarestky, S. L. Budko, P. C. Canfield, B. N. Harmon, A. I. Goldman, and R. J. McQueeney, Incommensurate Spin-Density Wave Order in Electron-Doped $\mathrm{BaFe}_{2} \mathrm{As}_{2}$ Superconductors, Phys. Rev. Lett. 106, 257001 (2011).

[22] H. Q. Luo, R. Zhang, M. Laver, Z. Yamani, M. Wang, X. Y. Lu, M. Y. Wang, Y. C. Chen, S. L. Li, S. Chang, J. W. Lynn, and P. Dai, Coexistence and Competition of the Short-Range Incommensurate Antiferromagnetic Order with the Superconducting State of $\mathrm{BaFe}_{2-x} \mathrm{Ni}_{x} \mathrm{As}_{2}$, Phys. Rev. Lett. 108, 247002 (2012).

[23] N. Qureshi, P. Steffens, Y. Drees, A. C. Komarek, D. Lamago, Y. Sidis, L. Harnagea, H.-J. Grafe, S. Wurmehl, B. Büchner, and M. Braden, Inelastic Neutron-Scattering Measurements of Incommensurate Magnetic Excitations on Superconducting LiFeAs Single Crystals, Phys. Rev. Lett. 108, 117001 (2012).

[24] L. Wang, F. Hardy, A. E. Böhmer, T. Wolf, P. Schweiss, and C. Meingast, Complex Phase Diagram of $\mathrm{Ba}_{1-x} \mathrm{Na}_{x} \mathrm{Fe}_{2} \mathrm{As}_{2}$ : A Multitude of Phases Striving for the Electronic Entropy, Phys. Rev. B 93, 014514 (2016).

[25] A. B. Vorontsov, M. G. Vavilov, and A. V. Chubukov, Interplay between Magnetism and Superconductivity in the Iron Pnictides, Phys. Rev. B 79, 060508(R) (2009).

[26] A. B. Vorontsov, M. G. Vavilov, and A. V. Chubukov, Superconductivity and Spin-Density Waves in Multiband Metals, Phys. Rev. B 81, 174538 (2010).

[27] D. J. Scalapino, A Common Thread: The Pairing Interaction for Unconventional Superconductors, Rev. Mod. Phys. 84, 1383 (2012).

[28] J. Paglione and R. L. Greene, High-Temperature Superconductivity in Iron-Based Materials, Nat. Phys. 6, 645 (2010).

[29] E. Wiesenmayer, H. Luetkens, G. Pascua, R. Khasanov, A. Amato, H. Potts, B. Banusch, H.-H. Klauss, and D. Johrendt, Microscopic Coexistence of Superconductivity and Magnetism in $\mathrm{Ba}_{1-x} \mathrm{~K}_{x} \mathrm{Fe}_{2} \mathrm{As}_{2}$, Phys. Rev. Lett. 107, 237001 (2011).

[30] S. Avci, O. Chmaissem, E. A. Goremychkin, S. Rosenkranz, J.-P. Castellan, D. Y. Chung, I. S. Todorov, J. A. Schlueter, H. Claus, M. G. Kanatzidis, A. Daoud-Aladine, D. Khalyavin, and R. Osborn, Magnetoelastic Coupling in the Phase
Diagram of $\mathrm{Ba}_{1-x} \mathrm{~K}_{x} \mathrm{Fe}_{2} \mathrm{As}_{2}$ as Seen via Neutron Diffraction, Phys. Rev. B 83, 172503 (2011).

[31] P. Materne, S. Kamusella, R. Sarkar, T. Goltz, J. Spehling, H. Maeter, L. Harnagea, S. Wurmehl, B. Bchner, H. Luetkens, C. Timm, and H.-H. Klauss, Coexistence of Superconductivity and Magnetism in $\mathrm{Ca}_{1-x} \mathrm{Na}_{x} \mathrm{Fe}_{2} \mathrm{As}_{2}$ : Universal Suppression of the Magnetic Order Parameter in 122 Iron Pnictides, Phys. Rev. B 92, 134511 (2015).

[32] N. Ni, M. E. Tillman, J.-Q. Yan, A. Kracher, S. T. Hannahs, S. L. Bud'ko, and P. C. Canfield, Effects of Co Substitution on Thermodynamic and Transport Properties and Anisotropic $\mathrm{H}_{c 2}$ in $\mathrm{Ba}\left(\mathrm{Fe}_{1-x} \mathrm{Co}_{x}\right)_{2} \mathrm{As}_{2}$ Single Crystals, Phys. Rev. B 78, 214515 (2008).

[33] S. Nandi, M. G. Kim, A. Kreyssig, R. M. Fernandes, D. K. Pratt, A. Thaler, N. Ni, S. L. Bud'ko, P. C. Canfield, J. Schmalian, R. J. McQueeney, and A. I. Goldman, Anomalous Suppression of the Orthorhombic Lattice Distortion in Superconducting $\mathrm{Ba}\left(\mathrm{Fe}_{1-x} \mathrm{Co}_{x}\right)_{2} \mathrm{As}_{2}$ Single Crystals, Phys. Rev. Lett. 104, 057006 (2010).

[34] B. Braunecker, G. I. Japaridze, J. Klinovaja, and D. Loss, Spin-Selective Peierls Transition in Interacting OneDimensional Conductors with Spin-Orbit Interaction, Phys. Rev. B 82, 045127 (2010).

[35] P. Bruno, V. K. Dugaev, and M. Taillefumier, Topological Hall Effect and Berry Phase in Magnetic Nanostructures, Phys. Rev. Lett. 93, 096806 (2004).

[36] G. Lawes and G. Srinivasan, Introduction to Magnetoelectric Coupling and Multiferroic Films, J. Phys. D 44, 243001 (2011).

[37] S. D. Ganichev and L. E. Golub, Interplay of Rashba/ Dresselhaus Spin Splittings Probed by Photogalvanic Spectroscopy-A Review, Phys. Status Solidi B 251, 1801 (2014).

[38] J. Sinova, S. O. Valenzuela, J. Wunderlich, C. H. Back, and T. Jungwirth, Spin Hall Effects, Rev. Mod. Phys. 87, 1213 (2015).

[39] X.-L. Qi and S.-C. Zhang, Topological Insulators and Superconductors, Rev. Mod. Phys. 83, 1057 (2011).

[40] T.-P. Choy, J. M. Edge, A. R. Akhmerov, and C. W. J. Beenakker, Majorana Fermions Emerging from Magnetic Nanoparticles on a Superconductor without Spin-Orbit Coupling, Phys. Rev. B 84, 195442 (2011).

[41] I. Martin and A.F. Morpurgo, Majorana Fermions in Superconducting Helical Magnets, Phys. Rev. B 85, 144505 (2012).

[42] M. Kjaergaard, K. Wölms, and K. Flensberg, Majorana Fermions in Superconducting Nanowires without SpinOrbit Coupling, Phys. Rev. B 85, 020503(R) (2012).

[43] S. Nadj-Perge, I. K. Drozdov, B. A. Bernevig, and A. Yazdani, Proposal for Realizing Majorana Fermions in Chains of Magnetic Atoms on a Superconductor, Phys. Rev. B 88, 020407(R) (2013).

[44] P. Kotetes, Classification of Engineered Topological Superconductors, New J. Phys. 15, 105027 (2013).

[45] S. Nakosai, Y. Tanaka, and N. Nagaosa, Two-Dimensional p-Wave Superconducting States with Magnetic Moments on a Conventional s-Wave Superconductor, Phys. Rev. B 88, 180503(R) (2013). 
[46] B. Braunecker and P. Simon, Interplay between Classical Magnetic Moments and Superconductivity in Quantum One-Dimensional Conductors: Toward a Self-Sustained Topological Majorana Phase, Phys. Rev. Lett. 111, 147202 (2013).

[47] J. Klinovaja, P. Stano, A. Yazdani, and D. Loss, Topological Superconductivity and Majorana Fermions in RKKY Systems, Phys. Rev. Lett. 111, 186805 (2013).

[48] M. M. Vazifeh and M. Franz, Self-Organized Topological State with Majorana Fermions, Phys. Rev. Lett. 111, 206802 (2013).

[49] F. Pientka, L. I. Glazman, and F. von Oppen, Topological Superconducting Phase in Helical Shiba Chains, Phys. Rev. B 88, 155420 (2013).

[50] J. Li, T. Neupert, B. A. Bernevig, and A. Yazdani, Manipulating Majorana Zero Modes on Atomic Rings with an External Magnetic Field, Nat. Commun. 7, 10395 (2016).

[51] M. Schecter, K. Flensberg, M. H. Christensen, B. M. Andersen, and J. Paaske, Self-Organized Topological Superconductivity in a Yu-Shiba-Rusinov Chain, Phys. Rev. B 93, 140503(R) (2016).

[52] M. H. Christensen, M. Schecter, K. Flensberg, B. M. Andersen, and J. Paaske, Spiral Magnetic Order and Topological Superconductivity in a Chain of Magnetic Adatoms on a Two-Dimensional Superconductor, Phys. Rev. B 94, 144509 (2016).

[53] K. Pöyhönen, A. Westström, J. Röntynen, and T. Ojanen, Majorana States in Helical Shiba Chains and Ladders, Phys. Rev. B 89, 115109 (2014).

[54] N. Sedlmayr, J. M. Aguiar-Hualde, and C. Bena, Flat Majorana Bands in Two-Dimensional Lattices with Inhomogeneous Magnetic Fields: Topology and Stability, Phys. Rev. B 91, 115415 (2015).

[55] D. Mendler, P. Kotetes, and G. Schön, Magnetic Order on a Topological Insulator Surface with Warping and ProximityInduced Superconductivity, Phys. Rev. B 91, 155405 (2015).

[56] W. Chen and A.P. Schnyder, Majorana Edge States in Superconductor-Noncollinear Magnet Interfaces, Phys. Rev. B 92, 214502 (2015).

[57] G. Yang, P. Stano, J. Klinovaja, and D. Loss, Majorana Bound States in Magnetic Skyrmions, Phys. Rev. B 93, 224505 (2016).

[58] S. Graser, A. F. Kemper, T. A. Maier, H.-P. Cheng, P. J. Hirschfeld, and D. J. Scalapino, Spin Fluctuations and Superconductivity in a Three-Dimensional Tight-Binding Model for $\mathrm{BaFe}_{2} \mathrm{As}_{2}$, Phys. Rev. B 81, 214503 (2010).

[59] H. Ikeda, R. Arita, and J. Kunes, Phase Diagram and Gap Anisotropy in Iron-Pnictide Superconductors, Phys. Rev. B 81, 054502 (2010).

[60] L. de Medici, in The Physics of Correlated Insulators, Metals, and Superconductors Modeling and Simulation, edited by E. Pavarini, E. Koch, R. Scalettar, and R. Martin (Verlag de Forschungszentrums Jülich, Jülich, 2017), Vol. 7, pp. 377-398.

[61] M. Yi, Y. Zhang, Z.-X. Shen, and D. Lu, Role of the Orbital Degree of Freedom in Iron-Based Superconductors, npj Quantum Mater. 2, 57 (2017).

[62] P. O. Sprau, A. Kostin, A. Kreisel, A. E. Böhmer, V. Taufour, P. C. Canfield, S. Mukherjee, P. J. Hirschfeld, B. M.
Andersen, and J. C. S. Davis, Discovery of Orbital-Selective Cooper Pairing in FeSe, Science 357, 75 (2017).

[63] A. Kreisel, B. M. Andersen, P. O. Sprau, A. Kostin, J. C. Séamus Davis, and P. J. Hirschfeld, Orbital Selective Pairing and Gap Structures of Iron-Based Superconductors, Phys. Rev. B 95, 174504 (2017).

[64] P. Zhang, K. Yaji, T. Hashimoto, Y. Ota, T. Kondo, K. Okazaki, Z. Wang, J. Wen, G. D. Gu, H. Ding, and S. Shin, Observation of Topological Superconductivity on the Surface of Iron-Based Superconductor, Science 360, 182 (2018).

[65] D. Wang, L. Kong, P. Fan, H. Chen, Y. Sun, S. Du, J. Schneeloch, R. D. Zhong, G. D. Gu, L. Fu, H. Ding, and H. Gao, Observation of Pristine Majorana Bound State in IronBased Superconductor, arXiv:1706.06074.

[66] H. J. Schulz, Incommensurate Antiferromagnetism in the Two-Dimensional Hubbard Model, Phys. Rev. Lett. 64, 1445 (1990).

[67] G. E. Volovik, The Universe in a Helium Droplet (Clarendon Press, Oxford, 2003).

[68] A. H. Castro Neto, F. Guinea, N. M. R. Peres, K. S. Novoselov, and A. K. Geim, The Electronic Properties of Graphene, Rev. Mod. Phys. 81, 109 (2009).

[69] S. Murakami, N. Nagaosa, and S.-C. Zhang, SU(2) NonAbelian Holonomy and Dissipationless Spin Current in Semiconductors, Phys. Rev. B 69, 235206 (2004).

[70] Y. Xia, D. Qian, D. Hsieh, L. Wray, A. Pal, H. Lin, A. Bansil, D. Grauer, Y. S. Hor, R. J. Cava, and M. Z. Hasan, Observation of a Large-Gap Topological-Insulator Class with a Single Dirac Cone on the Surface, Nat. Phys. 5, 398 (2009).

[71] L. Fu, Hexagonal Warping Effects in the Surface States of the Topological Insulator $\mathrm{Bi}_{2} \mathrm{Te}_{3}$, Phys. Rev. Lett. 103, 266801 (2009).

[72] A similar situation takes place for the surface states of warped topological insulators, cf. Ref. [71].

[73] D. Xiao, M.-C. Chang, and Q. Niu, Berry Phase Effects on Electronic Properties, Rev. Mod. Phys. 82, 1959 (2010).

[74] $\Theta$ is a symmetry of the IC magnetization profile alone since, due to the IC nature of $\boldsymbol{Q}_{1,2}$, the involved translations are not contained in the ones given by the crystalline periodicity of the FeSCs lattice. Nevertheless, as long as the low-energy properties of these itinerant magnets are primarily dictated by the Fermi-surface electrons, we expect $\Theta$ to be effectively present. This is because the electrons near the Fermi surface can be described by an effective continuum model which is unaware of the lattice constant.

[75] Notably, taking the commensurate limit for the magnetic profiles of DPHM and $\mathrm{SWC}_{4}$, without any further considerations, is ambiguous since $\left\{\mathrm{DPMH}, \mathrm{SWC}_{4}\right\} \rightarrow$ $\{\mathrm{CSDW}, \mathrm{SVC}\}$. This is due to the translational invariance of the system and our freedom to pick alternative spatial profiles other than the ones chosen in Sec. III. Nonetheless, by additionally demanding that the free-energy minimum varies continuously when taking this limit, Fig. 3(b) yields $\mathrm{DPMH} \rightarrow \mathrm{CSDW}$ and $\mathrm{SWC}_{4} \rightarrow \mathrm{SVC}$.

[76] D. F. Agterberg and H. Tsunetsugu, Dislocations and Vortices in Pair-Density-Wave Superconductors, Nat. Phys. 4, 639 (2008). 
[77] C. Castellani, C. R. Natoli, and J. Ranninger, Magnetic Structure of $\mathrm{V}_{2} \mathrm{O}_{3}$ in the Insulating Phase, Phys. Rev. B 18, 4945 (1978).

[78] A. M. Oleś, Antiferromagnetism and Correlation of Electrons in Transition Metals, Phys. Rev. B 28, 327 (1983).

[79] F. Wang, H. Zhai, Y. Ran, A. Vishwanath, and D.-H. Lee, Functional Renormalization-Group Study of the Pairing Symmetry and Pairing Mechanism of the FeAs-Based HighTemperature Superconductor, Phys. Rev. Lett. 102, 047005 (2009).

[80] C. Platt, W. Hanke, and R. Thomale, Functional Renormalization Group for Multi-Orbital Fermi Surface Instabilities., Adv. Phys. 62, 453 (2013).

[81] R.-Q. Xing, L. Classen, M. Khodas, and A. V. Chubukov, Competing Instabilities, Orbital Ordering, and Splitting of Band Degeneracies from a Parquet Renormalization Group Analysis of a Four-Pocket Model for Iron-Based Superconductors: Application to FeSe, Phys. Rev. B 95, 085108 (2017).

[82] D. Steffensen, M. H. Christensen, B. M. Andersen, and P. Kotetes, Topological Magnetic Superconductors (to be published).

[83] V. Cvetkovic and O. Vafek, Space Group Symmetry, SpinOrbit Coupling, and the Low-Energy Effective Hamiltonian for Iron-Based Superconductors, Phys. Rev. B 88, 134510 (2013).

[84] D. D. Scherer and B. M. Andersen, Spin-Orbit Coupling and Magnetic Anisotropy in Iron-Based Superconductors, Phys. Rev. Lett. 121, 037205 (2018).

[85] H. Eschrig and K. Koepernik, Tight-Binding Models for the Iron-Based Superconductors, Phys. Rev. B 80, 104503 (2009).

[86] N. Hao and J. Hu, Topological Phases in the Single-Layer FeSe, Phys. Rev. X 4, 031053 (2014).

[87] H. Luo, M. Wang, C. Zhang, X. Lu, L.-P. Regnault, R. Zhang, S. Li, J. Hu, and P. Dai, Spin Excitation Anisotropy as a Probe of Orbital Ordering in the Paramagnetic Tetragonal Phase of Superconducting $\mathrm{BaFe}_{1.904} \mathrm{Ni}_{0.096} \mathrm{As}_{2}$, Phys. Rev. Lett. 111, 107006 (2013).

[88] C. Zhang, M. Liu, Y. Su, L.-P. Regnault, M. Wang, G. Tan, T. Brückel, T. Egami, and P. Dai, Magnetic Anisotropy in Hole-Doped Superconducting $\mathrm{Ba}_{0.67} \mathrm{~K}_{0.33} \mathrm{Fe}_{2} \mathrm{As}_{2}$ Probed by Polarized Inelastic Neutron Scattering, Phys. Rev. B 87, 081101 (2013).

[89] N. Qureshi, C. H. Lee, K. Kihou, K. Schmalzl, P. Steffens, and M. Braden, Anisotropy of Incommensurate Magnetic Excitations in Slightly Overdoped $\mathrm{Ba}_{0.5} \mathrm{~K}_{0.5} \mathrm{Fe}_{2} \mathrm{As}_{2}$ Probed by Polarized Inelastic Neutron Scattering Experiments, Phys. Rev. B 90, 100502(R) (2014).

[90] Y. Song, H. R. Man, R. Zhang, X. Y. Lu, C. L. Zhang, M. Wang, G. T. Tan, L.-P. Regnault, Y. X. Su, J. Kang, R. M. Fernandes, and P.C. Dai, Spin Anisotropy due to Spin-Orbit Coupling in Optimally Hole-Doped $\mathrm{Ba}_{0.67} \mathrm{~K}_{0.33} \mathrm{Fe}_{2} \mathrm{As}_{2}$, Phys. Rev. B 94, 214516 (2016).

[91] For a weak SOC we can approach the problem using the already-obtained set of magnetic-order-parameter extrema of Eq. (1). However, if the SOC becomes strong, one should minimize the combination of Eqs. (1) and (29), that will generally lead to a new set of extrema.

[92] S. V. Borisenko, D. V. Evtushinsky, Z.-H. Liu, I. Morozov, R. Kappenberger, S. Wurmehl, B. Büchner, A. N. Yaresko, T. K. Kim, M. Hoesch, T. Wolf, and N. D. Zhigadlo, Direct Observation of Spin-Orbit Coupling in Iron-Based Superconductors, Nat. Phys. 12, 311 (2016).

[93] Note that the potential inclusion of SOC terms at quartic order would lower $T_{\text {mag }}^{(2)}$, and if they were sufficiently strong, they could even prevent the occurrence of the secondary transition. However, such a situation is beyond the scope and applicability of the present analysis.

[94] M. H. Christensen, P. P. Orth, B. M. Andersen, and R. M. Fernandes, Magnetic Phase Diagram of the Iron Pnictides in the Presence of Spin-Orbit Coupling: Frustration between $C_{2}$ and $C_{4}$ Magnetic Phases, Phys. Rev. B 98, 014523 (2018).

[95] M. N. Gastiasoro, I. Eremin, R. M. Fernandes, and B. M. Andersen, Scanning Tunneling Spectroscopy as a Probe of Multi-Q Magnetic States of Itinerant Magnets, Nat. Commun. 8, 14317 (2017).

[96] M. T. Mercaldo, M. Cuoco, and P. Kotetes, Magnetic-FieldInduced Topological Reorganization of a p-Wave Superconductor, Phys. Rev. B 94, 140503(R) (2016).

[97] Z. Wang, P. Zhang, Gang Xu, L. K. Zeng, H. Miao, X. Xu, T. Qian, H. Weng, P. Richard, A. V. Fedorov, H. Ding, X. Dai, and Z. Fang, Topological Nature of the $\mathrm{FeSe}_{0.5} \mathrm{Te}_{0.5}$ Superconductor, Phys. Rev. B 92, 115119 (2015).

[98] Z. F. Wang, H. Zhang, D. Liu, C. Liu, C. Tang, C. Song, Y. Zhong, J. Peng, F. Li, C. Nie, L. Wang, X. J. Zhou, X. Ma, Q. K. Xue, and F. Liu, Topological Edge States in a High-Temperature Superconductor $\mathrm{FeSe} / \mathrm{SrTiO}_{3}(001)$ Film, Nat. Mater. 15, 968 (2016).

[99] G. Xu, B. Lian, P. Tang, X.-L. Qi, and S.-C. Zhang, Topological Superconductivity on the Surface of Fe-Based Superconductors, Phys. Rev. Lett. 117, 047001 (2016). 